\title{
Adaptive Hybrid Intelligent Control for Uncertain Nonlinear Dynamical Systems
}

\author{
Chi-Hsu Wang, Senior Member, IEEE, Tsung-Chih Lin, Tsu-Tian Lee, Fellow, IEEE, and Han-Leih Liu
}

\begin{abstract}
A new hybrid direct/indirect adaptive fuzzy neural network (FNN) controller with state observer and supervisory controller for a class of uncertain nonlinear dynamic systems is developed in this paper. The hybrid adaptive FNN controller, the free parameters of which can be tuned on-line by observer-based output feedback control law and adaptive law, is a combination of direct and indirect adaptive FNN controllers. A weighting factor, which can be adjusted by the tradeoff between plant knowledge and control knowledge, is adopted to sum together the control efforts from indirect adaptive FNN controller and direct adaptive FNN controller. Furthermore, a supervisory controller is appended into the FNN controller to force the state to be within the constraint set. Therefore, if the FNN controller cannot maintain the stability, the supervisory controller starts working to guarantee stability. On the other hand, if the FNN controller works well, the supervisory controller will be deactivated. The overall adaptive scheme guarantees the global stability of the resulting closed-loop system in the sense that all signals involved are uniformly bounded. Two nonlinear systems, namely, inverted pendulum system and Chua's chaotic circuit, are fully illustrated to track sinusoidal signals. The resulting hybrid direct/indirect FNN control systems show better performances, i.e., tracking error and control effort can be made smaller and it is more flexible during the design process.
\end{abstract}

Index Terms-Adaptive control, fuzzy neural networks (FNNs), nonlinear systems, state observer, supervisory control.

\section{INTRODUCTION}

$\mathbf{M}$ OST current techniques for designing control systems are based on a good understanding of the plant under consideration and its environment. However, in a number of instances, the plant to be controlled is too complex and the basic physical processes in it are not fully understood. Hence, control design methods need to be augmented with an identification technique aimed at obtaining a progressively better understanding of the plant to be controlled. Adaptive control is a technique of applying some system identification techniques to obtain a model of the process and its environment from input/output experiment and using this model to design

Manuscript received August 3, 2001; revised January 18, 2002. This work was supported in part by the National Science Council, Taiwan, R.O.C., under Grant NSC91-2213-E009-067. This paper was recommended by Associate Editor C. T. Lin.

C.-H. Wang and H.-L. Liu are with the School of Microelectronic Engineering, Griffith University, Nathan, Brisbane Q4111, Australia (e-mail c.wang@me.gu.edu.au).

T.-C. Lin is with the Department of Electronic Engineering, Feng-Chia University, Taichung, Taiwan, R.O.C., and the School of Microelectronic Engineering, Griffith University, Nathan, Brisbane Q4111, Australia.

T.-T. Lee is with the Department of Electrical and Control Engineering, National Chiao-Tung University, Hsinchu 300, Taiwan, R.O.C.

Publisher Item Identifier S 1083-4419(02)05144-0. a controller. The adaptive control for feedback linearizable nonlinear systems is an approach to nonlinear control design that has attracted a great deal of interest in the nonlinear control community for at least a quarter of a century. By using feedback linearization [1]-[3], the nonlinear adaptive control problem is transformed into a linear adaptive control problem, then the linear control methods can be applied to acquire the desired performance. The adaptive control methodologies include direct adaptive control (DAC) and indirect adaptive control (IAC) algorithms [4]-[9].

Recently, an important adaptive fuzzy neural network (FNN) control system [4]-[14] has been proposed to incorporate with the expert information systematically, and the stability can be guaranteed by universal approximation theorem [15]. For systems with a high degree of nonlinear uncertainty, such as chemical process, aircraft, etc., they are very difficult to control using the conventional control theory. However, human operators can often successfully control them. Based on the fact that FNN logic systems are capable of uniformly approximating a nonlinear function over a compact set to any degree of accuracy, a globally stable adaptive FNN controller is defined as an FNN logic system equipped with an adaptation algorithm. Moreover, FNN is constructed from a collect of fuzzy IF-THEN rules using fuzzy logic principles, and the adaptation algorithm adjusts the free parameters of the FNN based on the numerical experiment data. Like the conventional adaptive control, the adaptive FNN control has direct and indirect FNN adaptive control categories [7], [8]. Direct adaptive FNN control has been discussed in [4] and [7], in which the adaptive FNN controller uses fuzzy logic systems as controller. Hence, linguistic fuzzy control rules can be directly incorporated into the controller. Also, indirect adaptive FNN control has been proposed in [4] and [7], in which the indirect FNN controller uses fuzzy descriptions to model the plant. Hence, fuzzy IF-THEN rules describing the plant can be directly incorporated into the indirect FNN controller.

Can these two adaptive FNN controllers be combined together to yield stable and robust adaptive control laws with supervisory controller? The answer is "yes." A hybrid direct/indirect adaptive FNN controller can be constructed by incorporating both fuzzy description and fuzzy control rules using a weighting factor $\alpha$ to sum together the control efforts from indirect adaptive FNN controller and direct adaptive FNN controller. The weighting factor $\alpha \in[0,1]$ can be adjusted by the tradeoff between plant knowledge and control knowledge. We let $\alpha=1$ if pure indirect adaptive FNN controller is required and $\alpha=0$ when pure direct adaptive FNN controller is chosen. If fuzzy control rules are more important and reliable than fuzzy 
descriptions of the plant, choose smaller $\alpha$; otherwise choose larger $\alpha$. In [4], [7], and [8], the full state must be assumed to be available for measurement. This assumption may not hold in practice because either the state variables are not accessible for direct connection or because sensing devices or transducers are not available. In this paper, our main objective is to create a technique for designing a state observer-based [12] hybrid direct/indirect adaptive FNN control for a class of uncertain nonlinear systems in which only the system output is measurable. Based on the Lyapunov synthesis approach, the free parameters of hybrid direct/indirect adaptive FNN controller can be tuned on-line by an observer-based output feedback control law and adaptive law. Also, a supervisory controller is designed to cascade with FNN controller. If the nonlinear system tends to unstable by the FNN controller, especially in the transient period, the supervisory controller will be activated to work with the FNN controller to stabilize the whole system. On the other hand, if the FNN controller works well, the supervisory controller will be deactivated. This will result in a smaller control effort (energy). Therefore, the overall adaptive scheme guarantees that the global stability of the resulting closed-loop system in the sense that all signals involved are uniformly bounded. We have successfully designed the FNN adaptive controllers with supervisory control to control the inverted pendulum and Chua's chaotic circuit [16] to track reference sinusoidal signals. The resulting hybrid direct/indirect FNN control systems show better performances, i.e., both tracking error and control effort can be made smaller.

This paper is organized as follows. Problem formulation is described in Section II. A brief description of the T-S FNN is presented in Section III. The observer-based hybrid direct/indirect FNN controller appended with a supervisory controller is constructed in Section IV. Simulation examples to demonstrate the performances of the proposed method are provided in Section V. Section VI lists the conclusions of the advocated design methodology.

\section{PROBLEM Formulation}

Consider the $n$ th-order nonlinear dynamical system of the form [1], [17]

$$
\begin{aligned}
\dot{x}_{1} & =x_{2} \\
\dot{x}_{2} & =x_{3} \\
& \ldots \\
\dot{x}_{n} & =f\left(x_{1}, x_{2}, \ldots, x_{n}\right)+g\left(x_{1}, x_{2}, \ldots, x_{n}\right) u+d \\
y & =x_{1}
\end{aligned}
$$

or equivalently the form

$$
\begin{aligned}
x^{(n)}=f(x, & \left.\dot{x}, \ldots, x^{(n-1)}\right) \\
& +g\left(x, \dot{x}, \ldots, x^{(n-1)}\right) u+d, \quad y=x
\end{aligned}
$$

where
$f$ and $g$ unknown but bounded functions;
$u \in R$ and $y \in R$ control input and output of the system, respectively;

Equation (1) [or (2)] is actually the Isidori-Byrnes canonical form [1], [17] for certain nonlinear systems. We consider only the nonlinear systems which can be represented by (1) or (2). The state space representation of (2) is expressed as

$$
\begin{aligned}
& \underline{\dot{x}}=A \underline{x}+B[f(\underline{x})+g(\underline{x}) u+d] \\
& y=C^{T} \underline{x}
\end{aligned}
$$

where

$$
\begin{aligned}
A & =\left[\begin{array}{lllllll}
0 & 1 & 0 & 0 & \cdots & 0 & 0 \\
0 & 0 & 1 & 0 & \cdots & 0 & 0 \\
\vdots & \vdots & \vdots & \vdots & \ddots & \vdots & \vdots \\
0 & 0 & 0 & 0 & \cdots & 0 & 1 \\
0 & 0 & 0 & 0 & \cdots & 0 & 0
\end{array}\right] \\
B & =\left[\begin{array}{c}
0 \\
0 \\
\vdots \\
0 \\
1
\end{array}\right], \quad C=\left[\begin{array}{c}
1 \\
0 \\
\vdots \\
0 \\
0
\end{array}\right]
\end{aligned}
$$

and $\underline{x}=\left[x_{1}, x_{2}, \ldots, x_{n}\right]^{T}=\left[x, \dot{x}, \ldots, x^{(n-1)}\right]^{T} \in R^{n}$ is a state vector where not all $x_{i}$ are assumed to be available for measurement. Only the system output $y$ is assumed to be measurable. In order for (2) to be controllable, it is required that $g(\underline{x}) \neq 0$ for $\underline{x}$ in a certain controllability region $U_{c} \subset R^{n}$. Without loss of generality, we assume that $0<g(\underline{x})<\infty$ for $\underline{x} \in U_{c}$. The control objective is to force the system output $y$ to follow a given bounded reference signal $y_{r}$, under the constraint that all signals involved must be bounded.

To begin with, the reference signal vector $\underline{y}_{r}$, the tracking error vector $\underline{\underline{e}}$, and estimation error vector $\underline{\hat{e}}$ will be defined as

$$
\begin{aligned}
\underline{y}_{r} & =\left[y_{r}, \dot{y}_{r}, \ldots, y_{r}^{(n-1)}\right]^{T} \in R^{n} \\
\underline{e} & =\underline{y}_{r}-\underline{x}=\left[e, \dot{e}, \ldots, e^{(n-1)}\right]^{T} \\
& \in R^{n}\left(e=y_{r}-x=y_{r}-y \in R\right) \\
\hat{\hat{e}} & =\underline{y}_{r}-\underline{\hat{x}}=\left[\hat{e}, \dot{\hat{e}}, \ldots, \hat{e}^{(n-1)}\right]^{T} \\
& \in R^{n}\left(\hat{e}=y_{r}-\hat{x} \in R\right)
\end{aligned}
$$

where $\underline{\hat{x}}$ and $\underline{\hat{e}}$ denote the estimates of $\underline{x}$ and $\underline{e}$, respectively.

If the functions $f(\underline{x})$ and $g(\underline{x})$ are known and the system is free of external disturbance $d$, then we can choose the controller $u^{*}$ to cancel the nonlinearity and design controller. In particular, let $\underline{k}_{c}=\left[k_{1}^{c}, k_{2}^{c}, \ldots, k_{n}^{c}\right]^{T} \in R^{n}$ be chosen such that all roots of the polynomial $p(s)=s^{n}+k_{n}^{c} s^{n-1}+\cdots+k_{1}^{c}$ are in the open-left half-plane and control law of the certainty equivalent controller is obtained as [8]

$$
u^{*}=\frac{1}{g(\underline{x})}\left[-f(\underline{x})+y_{r}^{(n)}+\underline{k}_{c}^{T} \underline{e}\right] .
$$

Substituting (5) into (2), we obtain the closed-loop system governed by

$$
e^{(n)}+k_{n}^{c} e^{(n-1)}+\cdots+k_{1}^{c} e=0
$$

where the main objective of the control is $\lim _{t \rightarrow \infty} e(t)=0$. However, $f(\underline{x})$ and $g(\underline{x})$ are unknown, the ideal controller (5) 
cannot be implemented, and not all system states $\underline{x}$ can be measured. We have to design an observer to estimate the state vector $\underline{x}$ in the following context.

\section{A. Observer-Based Hybrid Direct/Indirect FNN Controller With Supervisory Control Scheme}

Here, we will develop the observer-based hybrid direct/indirect FNN controller with supervisory control scheme. The overall control law is constructed as

$$
u=\alpha u_{I}(\underline{\hat{x}})+(1-\alpha) u_{D}\left(\underline{\hat{x}} \mid \underline{\theta}_{D}\right)+u_{S}(\underline{\hat{x}})
$$

where

$u_{I} \quad$ indirect FNN controller [see (8)];

$u_{D} \quad$ output of the Takagi-Sugeno (T-S)-based DAC FNN controller (described in Sections III and IV);

$u_{S} \quad$ supervisory control (described in Section IV) to force the state within the constraint set;

$\alpha \in[0,1]$ weighting factor.

If the plant knowledge is more important and reliable than the control knowledge, we should choose a larger $\alpha$; otherwise, a smaller $\alpha$ should be chosen. Since $\underline{x}$ cannot be available and $f(\underline{x})$ and $g(\underline{x})$ are unknown, we replace the functions $f(\underline{x})$, $g(\underline{x})$, and error vector $\underline{e}$ in (5) by estimation functions $\hat{f}(\underline{\hat{x}})$ and $\hat{g}(\underline{\hat{x}})$ (described in Section III), and $\underline{\hat{e}}$. The certainty equivalent controller can be rewritten as

$$
u^{*}=\frac{1}{g(\underline{x})}\left[-f(\underline{x})+y_{r}^{(n)}+\underline{k}_{c}^{T} \hat{\hat{e}}\right] .
$$

The indirect control law is written as

$$
u_{I}=\frac{1}{\hat{g}(\underline{\hat{x}})}\left[-\hat{f}(\underline{\hat{x}})+y_{r}^{(n)}+\underline{k}_{c}^{T} \underline{\hat{e}}\right] .
$$

Applying (6) and (7) to (3), and after some simple manipulations, we can obtain the error dynamic equation

$$
\begin{aligned}
\underline{\dot{e}}= & A \underline{e}-B \underline{k}_{c}^{T} \underline{\hat{e}}+B\left\{\alpha\left[\hat{f}(\underline{\hat{x}})-f(\underline{x})+(\hat{g}(\underline{\hat{x}})-g(\underline{x})) u_{I}\right]\right. \\
& \left.+(1-\alpha) g(\underline{x})\left(u^{*}-u_{D}\right)-g(\underline{x}) u_{S}-d\right\} \\
e_{1}= & C^{T} \underline{e}
\end{aligned}
$$

where $e_{1}=y_{r}-y=y_{r}-x_{1}$.

From (9), the following observer that estimates the state error vector $\underline{e}$ in $(9)$

$$
\begin{aligned}
\dot{\hat{\hat{e}}} & =A \underline{\hat{e}}-B \underline{k}_{c}^{T} \underline{\hat{e}}+\underline{k}_{o}\left(e_{1}-\hat{e}_{1}\right) \\
\hat{e}_{1} & =C^{T} \underline{\hat{e}}
\end{aligned}
$$

where $\underline{k}_{o}=\left[k_{n}^{\circ}, k_{n-1}^{o}, \ldots, k_{1}^{\circ}\right]^{T} \in R^{n}$ is the observer gain vector.

The observation errors are defined as: $\underline{\tilde{e}}=\underline{e}-\underline{\hat{e}}$ and $\tilde{e}_{1}=$ $e_{1}-\hat{e}_{1}$.

Subtracting (10) from (9), we can obtain the error dynamics

$$
\begin{aligned}
\dot{\underline{\tilde{e}}}= & \Lambda_{o} \underline{\underline{\tilde{e}}}+B\left\{\alpha\left[\hat{f}(\underline{\hat{x}})-f(\underline{x})+(\hat{g}(\underline{\hat{x}})-g(\underline{x})) u_{I}\right]\right. \\
& \left.+(1-\alpha) g(\underline{x})\left(u^{*}-u_{D}\right)\right\}-B g(\underline{x}) u_{S}-B d \\
\tilde{e}_{1}= & C^{T} \underline{\tilde{e}}
\end{aligned}
$$

where $\Lambda_{o}=A-\underline{k}_{o} C^{T}$. Since $\left(C, \Lambda_{0}\right)$ pair is observable, the observer gain vector $\underline{k}_{o}$ can be chosen such that the characteristic polynomial of $\Lambda_{0}$ is strictly Hurwitz (i.e., the roots of the closed-loop system are in the open-left half-plane) and we know that there exists a positive definite symmetric $n \times n$ matrix $P$ which satisfies the Lyapunov equation

$$
\Lambda_{o}^{T} P+P \Lambda_{o}=-Q
$$

where $Q$ is an arbitrary $n \times n$ positive definite matrix.

Let us rewrite (10) as

$$
\underline{\dot{\hat{e}}}=\hat{A} \underline{\hat{e}}+\underline{k}_{o} C^{T} \underline{\tilde{e}}
$$

where $\hat{A}=A-B \underline{k}_{c}^{T}$ is a strictly Hurwitz matrix. Therefore, there exists a positive definite symmetric $n \times n$ matrix $\hat{P}$ which satisfies the Lyapunov equation

$$
\hat{A}^{T} \hat{P}+\hat{P} \hat{A}=-\hat{Q}
$$

where $\hat{Q}$ is an arbitrary $n \times n$ positive definite matrix. Let $V_{\underline{\underline{\hat{e}}}}=$ $(1 / 2) \underline{\hat{e}}^{T} \hat{P} \underline{\hat{e}}$, then by using (13) and (14), we have

$$
\begin{aligned}
\dot{V}_{\underline{\hat{e}}} & =\frac{1}{2} \dot{\hat{e}}^{T} \hat{P} \underline{\hat{e}}+\frac{1}{2} \underline{\hat{e}}^{T} \hat{P} \underline{\hat{e}} \\
& =\frac{1}{2}\left\{\hat{A} \underline{\hat{e}}+\underline{k}_{o} C^{T} \underline{\tilde{e}}\right\}^{T} \hat{P} \underline{\hat{e}}+\frac{1}{2} \underline{\hat{e}}^{T} \hat{P}\left\{\hat{A} \underline{\hat{e}}+\underline{k}_{o} C^{T} \underline{\tilde{e}}\right\} \\
& =-\frac{1}{2} \underline{\hat{e}}^{T} \hat{Q} \underline{\hat{e}}+\underline{\hat{e}}^{T} \hat{P} \underline{k}_{o} C^{T} \underline{\tilde{e}} .
\end{aligned}
$$

Since $\hat{Q}$ and $\underline{k}_{O}$ are determined by the designer, we can choose $\hat{Q}$ and $\underline{k}_{o}$, such that $\dot{V}_{\hat{\underline{e}}} \leq 0$. Hence, $V_{\underline{\underline{e}}}$ is a bounded function and there exists a constant value $\bar{V}_{\underline{\underline{\hat{e}}}}$, such that $V_{\underline{\underline{\hat{e}}}} \leq \bar{V}_{\underline{\underline{\hat{e}}}}$.

\section{The TAKAGI-SUGENO (T-S) FNN SYSTEMS}

Fuzzy logic systems address the imprecision of the input and output variables directly by defining them with fuzzy numbers (and fuzzy sets) that can be expressed in linguistic terms (e.g., small, medium, and large). The basic configuration the T-S FNN system [18]-[22] includes a fuzzy rule base, which consists of a collection of fuzzy IF-THEN rules in the following form:

$$
\begin{aligned}
& R^{(l)}: \operatorname{IF~} x_{1} \text { is } F_{1}^{l}, \text { and } \ldots, \text { and } x_{n} \text { is } F_{n}^{l}, \\
& \quad \text { THEN } y_{l}=q_{0}^{l}+q_{1}^{l} x_{1}+\cdots+q_{n}^{l} x_{n}=\underline{\theta}_{l}^{T}\left[1 \underline{x}^{T}\right]^{T}
\end{aligned}
$$

where $F_{i}^{l}$ are fuzzy sets and $\underline{\theta}_{l}^{T}=\left[q_{0}^{l}, q_{1}^{l}, \ldots, q_{n}^{l}\right]$ is a vector of the adjustable factors of the consequence part of the fuzzy rule. Furthermore, $y_{l}$ is a linguistic variable, and a fuzzy inference engine to combine the fuzzy IF-THEN rules in the fuzzy rule base into a mapping from an input linguistic vector $\underline{x}^{T}=$ $\left[x_{1}, x_{2}, \ldots, x_{n}\right] \in R^{n}$ to an output variable $y \in R$. Let $M$ be the number of the fuzzy IF-THEN rules. The output of the fuzzy logic systems with central average defuzzifier, product inference, and singleton fuzzifier can be expressed as

$$
y(\underline{x})=\frac{\sum_{l=1}^{M} v^{l} \cdot y_{l}}{\sum_{l=1}^{M} v^{l}}=\frac{\sum_{l=1}^{M} v^{l} \cdot \underline{\theta}_{l}^{T}\left[1 \underline{x}^{T}\right]}{\sum_{l=1}^{M} v^{l}}
$$




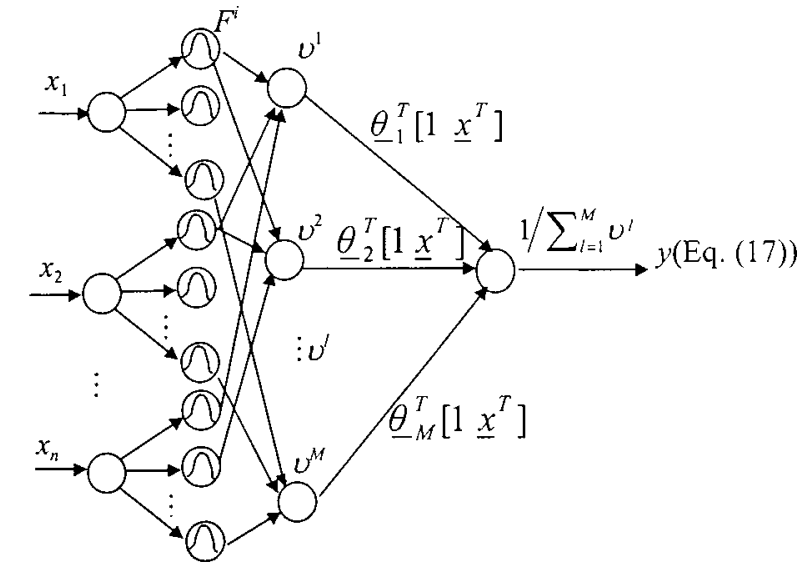

Layer I Layer II Layer III Layer IV

Premise Consequence

Fig. 1. Configuration of the T-S FNNs.

where $\mu_{F_{i}^{l}}\left(x_{i}\right)$ is the membership function value of the fuzzy variable $x_{i}$ and $v^{l}=\prod_{i=1}^{n} \mu_{F_{i}^{l}}\left(x_{i}\right)$ is the true value of the $l$ th implication. Equation (17) can be rewritten as

$$
y(\underline{x})=\underline{\theta}^{T} \underline{\psi}(\underline{x})
$$

where $\underline{\theta}^{T}=\left[\underline{\theta}_{1}^{T} \underline{\theta}_{2}^{T} \cdots \underline{\theta}_{M}^{T}\right]$ is an adjustable parameter vector and $\psi^{T}(\underline{x})=\left[\psi^{1}(\underline{x}), \psi^{2}(\underline{x}), \ldots, \psi^{M}(\underline{x})\right]$ is a fuzzy basis function vector defined as

$$
\psi^{l}(\underline{x})=\frac{v^{l}\left[1 \underline{x}^{T}\right]}{\sum_{l=1}^{M} v^{l}} .
$$

When the inputs are fed into the T-S FNN, the true value $v^{l}$ of the $l$ th implication is computed. Applying the common defuzzification strategy, the output of the NNs expressed as (17) is pumped out. The overall configuration of the T-S FNN is shown in Fig. 1.

Based on the universal approximation theorem [15], the aforementioned fuzzy logic system is capable of uniformly approximating any well-defined nonlinear function over a compact set $U_{c}$ to any degree of accuracy. It is also straightforward to show that a multi-output system can always be approximated by a group of single-output approximation systems.

\section{HYBRID DIRECT/INDIRECT ADAPTIVE FNN CONTROLLER WITH OBSERVER AND SUPERVISORY CONTROLLER}

An adaptive fuzzy system is a fuzzy logic system equipped with a training algorithm to maintain a consistent performance under plant uncertainties. The most important advantage of the adaptive FNN control over conventional adaptive control is that adaptive FNN controllers are capable of incorporating linguistic fuzzy information from a human operator, whereas the conventional adaptive controller is not. The adaptive FNN control is divided into two categories. One is called the indirect adaptive
FNN control and the other is called the direct adaptive FNN control [7], [8]. An adaptive FNN controller that uses fuzzy logic systems as a model of the plant is an indirect adaptive FNN controller. An adaptive FNN controller that directly uses fuzzy logic systems as controller is a direct adaptive FNN controller. Therefore, the indirect adaptive FNN controller can incorporate fuzzy descriptions but cannot incorporate fuzzy control rules. On the other hand, the direct adaptive FNN controller can incorporate fuzzy control rules but cannot incorporate fuzzy descriptions. In this section, we will develop the hybrid direct/indirect adaptive FNN controller that can incorporate linguistic information and design an adaptive law for the adjustable parameters in the controller, such that the closed loop output $y(t)$ follows the reference output $y_{r}(t)$.

Let us replace $\hat{f}(\underline{\hat{x}}), \hat{g}(\underline{\hat{x}})$, and $u_{D}(\underline{\hat{x}})$ in (11) by the fuzzy logic system $\hat{f}(\underline{\hat{x}} \mid \underline{\theta} f), \hat{g}\left(\underline{\hat{x}} \mid \underline{\theta}_{g}\right)$, and $u_{D}\left(\underline{\hat{x}} \mid \underline{\theta}_{D}\right)$, respectively. Therefore, the error dynamics (11) can be rewritten as

$$
\begin{aligned}
\underline{\dot{\tilde{e}}}= & \Lambda_{o} \underline{\tilde{e}}+B\left\{\alpha\left[\hat{f}\left(\underline{\hat{x}} \mid \underline{\theta}_{f}\right)-f(\underline{x})+\left(\hat{g}\left(\underline{\hat{x}} \mid \underline{\theta}_{g}\right)-g(\underline{x})\right) u_{I}\right]\right. \\
& +(1-\alpha) g(\underline{x})\left(u^{*}-u_{D}\left(\underline{\hat{x}} \mid \underline{\theta}_{D}\right)\right\} \\
& -B g(\underline{x}) u_{S}-B d .
\end{aligned}
$$

Let $V_{\underline{\tilde{e}}}=(1 / 2) \underline{\tilde{e}}^{T} P \underline{\tilde{e}}$, then using (12) and (20) we have

$$
\begin{aligned}
\dot{V}_{\underline{\tilde{e}}}= & \frac{1}{2} \underline{\tilde{e}}^{T} P \underline{\tilde{e}}+\frac{1}{2} \underline{\tilde{e}}^{T} P \underline{\dot{e}} \\
= & -\frac{1}{2} \underline{\tilde{e}}^{T} Q \underline{\tilde{e}}+\alpha \underline{\tilde{e}}^{T} P B \\
& \times\left[\hat{f}(\underline{\hat{x}} \mid \underline{\theta} f)-f(\underline{x})+(\hat{g}(\underline{\hat{x}} \mid \underline{\theta} g)-g(\underline{x})) u_{I}\right] \\
& +\underline{\tilde{e}}^{T} P B(1-\alpha) g(\underline{x})\left(u^{*}-u_{D}\left(\underline{\hat{x}} \mid \underline{\theta}_{D}\right)\right) \\
& -\underline{\tilde{e}}^{T} P B g(\underline{x}) u_{S}-\underline{\tilde{e}}^{T} P B d \\
\leq & -\frac{1}{2} \underline{\tilde{e}}^{T} Q \underline{\tilde{e}}+\left|\underline{\tilde{e}}^{T} P B\right| \\
& \times\left\{\alpha\left[\left|\hat{f}\left(\underline{\hat{x}} \mid \underline{\theta}_{f}\right)\right|+|f(\underline{x})|+\left|\hat{g}\left(\underline{\hat{x}} \mid \underline{\theta}_{g}\right) u_{I}\right|+\left|g(\underline{x}) u_{I}\right|\right]\right. \\
& \left.\quad+(1-\alpha)\left|g(\underline{x}) u^{*}\right|+\left|g(\underline{x}) u_{D}\left(\underline{\hat{x}} \mid \underline{\theta}_{D}\right)\right|+|d|\right\} \\
& -\underline{\tilde{e}}^{T} P B g(\underline{x}) u_{S} .
\end{aligned}
$$

In order to design $u_{S}$ such that $\dot{V}_{\tilde{\underline{e}}} \leq 0$, we need the following assumption.

Assumption I: We can determine functions $f^{U}(\underline{x}), g^{U}(\underline{x})$, and $g_{L}(\underline{x})$ such that $|f(\underline{x})| \leq f^{U}(\underline{x}) \approx f^{U}(\underline{\hat{x}})$ and $g_{L}(\underline{\hat{x}}) \approx$ $g_{L}(\underline{x}) \leq g(\underline{x}) \leq g^{U}(\underline{x}) \approx g^{U}(\underline{\hat{x}})$ for $\underline{x} \in U_{c}$, where $f^{U}(\underline{x}) \approx$ $f^{U}(\underline{\hat{x}})<\infty, g^{U}(\underline{x}) \approx g^{U}(\underline{\hat{x}})<\infty$, and $g_{L}(\underline{x}) \approx g_{L}(\underline{\hat{x}})>0$ for $\underline{x} \in U_{c}$. This is due to the fact that we can choose $\underline{k}_{o}$ in (10) to let $\underline{x} \approx \underline{\hat{x}}$. Furthermore, external disturbance is bounded, i.e., $|d| \leq d_{m}$ where $d_{m}$ is the upper bound of noise $d$.

From Assumption I, and by observing (21), we choose the supervisory control $u_{S}$ as

$$
\begin{aligned}
u_{S}=I^{*} & \operatorname{sgn}\left(\underline{\tilde{e}}^{T} P B\right) \frac{1}{g_{L}(\underline{x})} \\
\times & {\left[|\hat{f}(\underline{\hat{x}} \mid \underline{\theta} f)|+2 f^{U}(\underline{x})+\left|y_{r}^{(n)}\right|+\left|\underline{k}_{c}^{T} \underline{\hat{e}}\right|\right.} \\
& \left.+g^{U}(\underline{x})\left(\left|u_{I}\right|+\left|u_{D}\right|\right)+\left|\hat{g}\left(\underline{\hat{x}} \mid \underline{\theta}_{g}\right) u_{I}\right|+d_{m}\right]
\end{aligned}
$$


where $I^{*}=1$ if $V_{\tilde{e}}>\bar{V}$ (which is a constant chosen by the designer), $I^{*}=0$ if $V_{\tilde{e}} \leq \bar{V}$, and $\operatorname{sgn}(\tau)=1(-1)$ if $\tau \geq$ $0(<0)$. Considering the case $V_{\underline{\underline{e}}}>\bar{V}$ and substituting (22) into (21), we obtain

$$
\begin{aligned}
\dot{V}_{\underline{\underline{e}} \leq} \leq & -\frac{1}{2} \underline{\tilde{e}}^{T} Q \underline{\tilde{e}}+\left|\underline{\tilde{e}}^{T} P B\right| \\
& \times\left\{\alpha\left[\left|\hat{f}\left(\underline{\hat{x}} \mid \underline{\theta}_{f}\right)\right|+|f(\underline{x})|+\left|\hat{g}\left(\underline{\hat{x}} \mid \underline{\theta}_{g}\right) u_{I}\right|+\left|g(\underline{x}) u_{I}\right|\right]\right. \\
& \left.+(1-\alpha)\left|g(\underline{x}) u^{*}\right|+\left|g(\underline{x}) u_{D}(\underline{\hat{x}} \mid \underline{\theta} D)\right|+|d|\right\} \\
& -\underline{\hat{e}}^{T} P B g(\underline{x}) u_{S} \\
\leq & -\frac{1}{2} \underline{\tilde{e}}^{T} Q \underline{\underline{e}}+\left|\underline{\hat{e}}^{T} P B\right|\left\{(1-\alpha)\left|g(\underline{x}) u^{*}\right|\right. \\
& \left.-\frac{g(\underline{x})}{g_{L}(\underline{x})}\left(f^{U}(\underline{x})+\left|y_{r}^{(n)}\right|+\left|\underline{k}_{c}^{T} \underline{\hat{e}}\right|\right)\right\} \\
\leq & -\frac{1}{2} \underline{\tilde{e}}^{T} Q \underline{\tilde{e}}+\left|\underline{\tilde{e}}^{T} P B\right| \\
& \times\left\{(1-\alpha)|g(\underline{x})|\left(|f(\underline{x})|+\left|y_{r}^{(n)}\right|+\left|\underline{k}_{c}^{T} \underline{\hat{e}}\right|\right)\right. \\
& \left.-\frac{g(\underline{x})}{g_{L}(\underline{x})}\left(f^{U}(\underline{x})+\left|y_{r}^{(n)}\right|+\left|\underline{k}_{c}^{T} \underline{\hat{e}}\right|\right)\right\} \\
\leq & -\frac{1}{2} \underline{\tilde{e}}^{T} Q \underline{\tilde{e}} \leq 0 .
\end{aligned}
$$

Therefore, we always have $V_{\tilde{e}} \leq \bar{V}$, by using the supervisory control $u_{S}$ [see (22)]. Because $P>0$, the bound of $V_{\tilde{e}}$ implies the bound of $\underline{\tilde{e}}$, which in turn implies the bound of $\underline{\hat{e}}$. Moreover, it implies the bound of $\underline{\hat{x}}$. It is obvious that the supervisory control $u_{S}$ is nonzero when $V_{\tilde{\underline{e}}}$ is greater than a positive value $\bar{V}$. Therefore, if the closed-loop system with the fuzzy controller $u$ as

$$
\begin{aligned}
u=\frac{\alpha}{\hat{g}\left(\underline{\hat{x}} \mid \underline{\theta}_{g}\right)}\left[-\hat{f}\left(\underline{\hat{x}} \mid \underline{\theta}_{f}\right)+y_{r}^{(n)}+\underline{k}_{c}^{T} \underline{\hat{e}}\right] \\
+(1-\alpha) u_{D}\left(\underline{\hat{x}} \mid \underline{\theta}_{D}\right)+u_{S}
\end{aligned}
$$

works well in the sense that the error is not too large, i.e., $V_{\tilde{\underline{e}}} \leq$ $\bar{V}$, then the supervisory control $u_{s}$ is zero. On the other hand, if the system tends to diverge, i.e., $V_{\tilde{e}}>\bar{V}$, then the supervisory control $u_{S}$ begins to operate to force $V_{\tilde{e}} \leq \bar{V}$.

We replace $\hat{f}\left(\underline{\hat{x}} \mid \underline{\theta}_{f}\right), \hat{g}\left(\underline{\hat{x}} \mid \underline{\theta}_{g}\right)$, and $u_{D}\left(\underline{\hat{x}} \mid \underline{\theta}_{D}\right)$ in specific fuzzy logic systems as (18), i.e.,

$$
\begin{aligned}
\hat{f}\left(\underline{\hat{x}} \mid \underline{\theta}_{f}\right) & =\underline{\xi}^{T}(\underline{\hat{x}}) \underline{\theta}_{f} \\
\hat{g}\left(\underline{\hat{x}} \mid \underline{\theta}_{g}\right) & =\underline{\xi}^{T}(\underline{\hat{x}}) \underline{\theta}_{g} \\
u_{D}\left(\underline{\hat{x}} \mid \underline{\theta}_{D}\right) & =\underline{\eta}^{T}(\underline{\hat{x}}) \underline{\theta}_{D}
\end{aligned}
$$

where $\underline{\xi}(\underline{\hat{x}})$ is a vector of fuzzy base, and $\underline{\theta}_{f}$ and $\underline{\theta}_{g}$ are the corresponding parameters of fuzzy logic systems. Also, $\eta(\underline{\hat{x}})$ is a vector of fuzzy base, and $\underline{\theta} \underline{D}_{D}$ is the corresponding parameters of fuzzy logic systems. In order to adjust the parameters in the fuzzy logic systems, we have to derive adaptive laws. Hence, the optimal parameter estimations $\underline{\theta}_{f}^{*}, \underline{\theta}_{g}^{*}$, and $\underline{\theta}_{D}^{*}$ are defined as

$$
\begin{aligned}
& \underline{\theta}_{f}^{*}=\arg \min _{\underline{\theta}_{f} \in \Omega_{f}}\left[\sup _{\underline{\underline{x}} \in \Omega_{\underline{\underline{x}}}, \underline{x} \in \Omega_{\underline{\underline{x}}}}\left|\hat{f}\left(\underline{\hat{x}} \mid \underline{\theta}_{f}\right)-f(\underline{x})\right|\right] \\
& \underline{\theta}_{g}^{*}=\arg \min _{\underline{\theta}_{g} \in \Omega_{g}}\left[\sup _{\underline{\underline{\hat{x}}} \in \Omega_{\underline{\underline{\hat{x}}}}, \underline{x} \in \Omega_{\underline{x}}}\left|\hat{g}\left(\underline{\hat{x}} \mid \underline{\theta}_{g}\right)-g(\underline{x})\right|\right]
\end{aligned}
$$

and

$$
\underline{\theta}_{D}^{*}=\arg \min _{\underline{\theta}_{D} \in \Omega_{D}}\left[\sup _{\underline{\hat{x}} \in \Omega_{\underline{\underline{x}}}, \underline{x} \in \Omega_{\underline{\underline{x}}}}\left|u^{*}(\underline{x})-u\left(\underline{\hat{x}} \mid \underline{\theta}_{D}\right)\right|\right]
$$

where $\Omega_{f}, \Omega_{g}, \Omega_{D}, \Omega_{\underline{\underline{x}}}$, and $\Omega_{\underline{x}}$ are compact sets of suitable bounds on $\underline{\theta}_{f}, \underline{\theta}_{g}, \underline{\theta}_{D}, \underline{\hat{x}}$, and $\underline{\underline{x}}$, respectively, and they are defined as $\Omega_{f}=\left\{\underline{\theta}_{f}|| \underline{\theta}_{f} \mid \leq M_{f}\right\}, \Omega_{g}=\left\{\underline{\theta}_{g}|| \underline{\theta}_{g} \mid \leq M_{g}\right\}$, $\Omega_{D}=\left\{\underline{\theta}_{D}|| \underline{\theta} D \mid \leq M_{D}\right\}, \Omega_{\underline{\underline{x}}}=\left\{\underline{\hat{x}}|| \underline{\hat{x}} \mid \leq M_{\hat{\underline{x}}}\right\}$, and $\Omega_{\underline{x}}=\left\{\underline{x}|| \underline{x} \mid \leq M_{\underline{x}}\right\}$, where $M_{f}, M_{g}, M_{D}, M_{\underline{\underline{x}}}$, and $M_{\underline{x}}$ are positive constants.

Define the minimum approximation errors as

$$
\begin{aligned}
\omega=\alpha\left[\hat{f}\left(\underline{\hat{x}} \mid \underline{\theta}_{f}^{*}\right)-\right. & \left.f(\underline{x})+\left(\hat{g}\left(\underline{\hat{x}} \mid \underline{\theta}_{g}^{*}\right)-g(\underline{x})\right) u_{I}\right] \\
& \left.+(1-\alpha) g(\underline{x})\left(u^{*}-u_{D(\underline{\hat{x}}} \mid \underline{\theta}_{D}^{*}\right)\right)-d .
\end{aligned}
$$

The error dynamics (20) can be expressed as

$$
\begin{aligned}
& \dot{\tilde{\tilde{e}}}=\Lambda_{o} \underline{\underline{e}}-B g(\underline{x}) u_{S}+B \\
& \times\left\{\alpha\left[\hat{f}\left(\underline{\hat{x}} \mid \underline{\theta}_{f}\right)-\hat{f}\left(\underline{\hat{x}} \mid \underline{\theta}_{f}^{*}\right)+\left(\hat{g}\left(\underline{\hat{x}} \mid \underline{\theta}_{g}\right)-g\left(\underline{\hat{x}} \mid \underline{\theta}_{g}^{*}\right)\right) u_{I}\right]\right. \\
&\left.\quad(1-\alpha) g(\underline{x})\left(u_{D}\left(\underline{\hat{x}} \mid \underline{\theta}_{D}\right)-u_{D}\left(\underline{\hat{x}} \mid \underline{\theta}_{D}^{*}\right)\right)+\omega\right\} . \quad \text { (32) }
\end{aligned}
$$

Substituting (25)-(27) into (32), the above equation can be rewritten as

$$
\begin{aligned}
\underline{\dot{\tilde{e}}}=\Lambda_{o} \underline{\tilde{e}}-B g(\underline{x}) u_{S}+ & B \alpha\left[\underline{\xi}^{T}(\underline{\hat{x}}) \underline{\tilde{\theta}}_{f}+\underline{\xi}^{T}(\underline{\hat{x}}) \underline{\tilde{\theta}}_{g} u_{I}\right] \\
& -B(1-\alpha) g(\underline{x}) \underline{\eta}^{T}(\underline{\hat{x}}) \underline{\tilde{\theta}}_{D}+B \omega
\end{aligned}
$$

where $\underline{\tilde{\theta}}_{f}=\underline{\theta}_{f}-\underline{\theta}_{f}^{*}, \underline{\tilde{\theta}}_{g}=\underline{\theta}_{g}-\underline{\theta}_{g}^{*}$, and $\underline{\tilde{\theta}}_{D}=\underline{\theta}_{D}-\underline{\theta}_{D}^{*}$. Now consider the Lyapunov function

$$
V=\frac{1}{2} \underline{\tilde{e}}^{T} P \underline{\tilde{e}}+\frac{\alpha}{2 \gamma_{1}} \underline{\tilde{\theta}}_{f}^{T} \underline{\tilde{\theta}}_{f}+\frac{\alpha}{2 \gamma_{2}} \underline{\tilde{\theta}}_{g}^{T} \underline{\tilde{\theta}}_{g}+\frac{(1-\alpha)}{2 \gamma_{3}} \underline{\tilde{\theta}}_{D}^{T} \underline{\tilde{\theta}}_{D} \text {. }
$$

The time derivative of $V$ is

$$
\begin{aligned}
\dot{V}=\frac{1}{2} \dot{\tilde{e}}^{T} P \underline{\underline{e}}+\frac{1}{2} \underline{\underline{e}}^{T} P \underline{\dot{\tilde{e}}}+\frac{\alpha}{\gamma_{1}} \underline{\tilde{\tilde{\theta}}}_{f}^{T} \underline{\tilde{\theta}}_{f} & +\frac{\alpha}{\gamma_{2}} \underline{\tilde{\tilde{\theta}}}_{g}^{T} \underline{\tilde{\theta}}_{g} \\
& +\frac{(1-\alpha)}{\gamma_{3}} \underline{\tilde{\hat{\theta}}}_{D}^{T} \underline{\tilde{\theta}}_{D} .
\end{aligned}
$$

Since $\underline{\tilde{\theta}}_{f}=\underline{\dot{\theta}}_{f}, \underline{\tilde{\theta}}_{g}=\underline{\dot{\theta}}_{g}$, and $\underline{\tilde{\theta}}_{D}=\underline{\dot{\theta}}_{D}$, and by using (12) and (34), (35) can be rewritten as

$$
\begin{aligned}
& \dot{V}=\frac{1}{2}\left\{\underline{\tilde{e}}^{T} \Lambda_{o}^{T} P \underline{\tilde{e}}-u_{S} g(\underline{x}) B^{T} P \underline{\tilde{e}}+\alpha \underline{\tilde{\theta}}{ }_{f}^{T} \underline{\xi}(\underline{\hat{x}}) B^{T} P \underline{\tilde{e}}\right. \\
& +\alpha u_{I} \underline{\tilde{\theta}}_{g}^{T} \underline{\xi}(\underline{\hat{x}}) B^{T} P \underline{\tilde{e}}-(1-\alpha) \underline{\tilde{\theta}}_{D}^{T} \underline{\eta}(\underline{\hat{x}}) g(\underline{x}) B^{T} P \underline{\tilde{e}} \\
& +\omega B^{T} P \underline{\tilde{e}}+\underline{\tilde{e}}^{T} P \Lambda_{o} \underline{\tilde{e}}-\underline{\tilde{e}}^{T} P B g(\underline{x}) u_{S} \\
& +\alpha \underline{\tilde{e}}^{T} P B \underline{\xi}^{T}(\underline{\hat{x}}) \underline{\tilde{\theta}}_{f}+\alpha \underline{\underline{e}}^{T} P B \underline{\xi}^{T}(\underline{\hat{x}}) \underline{\tilde{\theta}}_{g} u_{I} \\
& \left.-(1-\alpha) \underline{\tilde{e}}^{T} P B g(\underline{x}) \underline{\eta}^{T}(\underline{\hat{x}}) \underline{\tilde{\theta}}_{D}+\underline{\tilde{e}}^{T} P B \omega\right\} \\
& +\frac{\alpha}{\gamma_{1}} \dot{\tilde{\tilde{\theta}}}_{f}^{T} \tilde{\underline{\theta}}_{f}+\frac{\alpha}{\gamma_{2}} \dot{\tilde{\tilde{\theta}}}_{g}^{T} \tilde{\underline{\theta}}_{g}+\frac{(1-\alpha)}{\gamma_{3}} \underline{\tilde{\tilde{\theta}}}_{D}^{T} \underline{\tilde{\theta}}_{D} \\
& =-\frac{1}{2} \underline{\tilde{e}}^{T} Q \underline{\tilde{e}}-\underline{\tilde{e}}^{T} P B g(\underline{x}) u_{S}+\underline{\tilde{e}}^{T} P B \omega \\
& +\frac{\alpha}{\gamma_{1}}\left(\underline{\dot{\theta}}_{f}^{T}+\gamma_{1} \underline{\tilde{e}}^{T} P B \underline{\xi}^{T}(\underline{\hat{x}})\right) \underline{\tilde{\theta}}_{f} \\
& +\frac{\alpha}{\gamma_{2}}\left(\underline{\dot{\theta}}_{g}^{T}+\gamma_{2} \underline{\underline{e}}^{T} P B \underline{\xi}^{T}(\underline{\hat{x}}) u_{I}\right) \underline{\tilde{\theta}}_{g} \\
& +\frac{(1-\alpha)}{\gamma_{3}}\left(\underline{\dot{\theta}}_{D}^{T}-\gamma_{3} \underline{\tilde{e}}^{T} P B g(\underline{x}) \underline{\eta}^{T}(\underline{\hat{x}})\right) \underline{\tilde{\theta}}_{D} .
\end{aligned}
$$


According to (22) and $g(\underline{x})>0$, we have $g(\underline{x}) \underline{e}^{T} P B u_{S} \geq 0$. If the adaptive laws are chosen as

$$
\begin{aligned}
\underline{\dot{\theta}}_{f} & =-\gamma_{1} \underline{\xi}(\underline{\hat{x}}) B^{T} P \underline{\tilde{e}} \\
\underline{\dot{\theta}}_{g} & =-\gamma_{2} \underline{\xi}(\underline{\hat{x}}) B^{T} P \underline{\tilde{e}} u_{I} \\
\underline{\dot{\theta}}_{D} & \left.=\gamma_{3} \underline{\eta} \underline{\hat{x}}\right) g(\underline{x}) B^{T} P \underline{\tilde{e}} .
\end{aligned}
$$

Substituting (37)-(39) into (36), we have

$$
\dot{V} \leq-\frac{1}{2} \underline{\tilde{e}}^{T} Q \underline{\tilde{e}}+\underline{\tilde{e}}^{T} P B \omega .
$$

Since the term $\underline{\tilde{e}}^{T} P B \omega$ is of the order of the minimum approximation error, this is the best we can hope to obtain. If $\omega=0$, from (40) we have

$$
\dot{V}_{\underline{\tilde{e}}} \leq-\frac{1}{2} \underline{\tilde{e}}^{T} Q \underline{\tilde{e}} \leq 0 \text {. }
$$

If $\omega$ is not equal to zero, we can expect $\omega$ to be small based on the universal approximation theorem. From (28) to (30), the constraint sets $\Omega_{f}, \Omega_{g}$, and $\Omega_{D}$ of the optimal parameters $\underline{\theta}_{f}^{*}$, $\underline{\theta}_{g}^{*}$, and $\underline{\theta}_{D}^{*}$, respectively, if we can constrain $\underline{\theta}_{f}, \underline{\theta}_{g}$, and $\underline{\theta}_{D}$ within the sets, then $u$ in (24) and $u_{S}$ in (22) will be bounded due to the fact that, in this case, $\hat{f}, \hat{g}$, and $u_{D}$ are bounded, and it should be reminded that $\underline{\tilde{e}}$ is bounded because of the supervisory control $u_{S}$. Obviously, the adaptive laws in (37)-(39) are unable to guarantee that $\underline{\theta}_{f} \in \Omega_{f}, \underline{\theta}_{g} \in \Omega_{g}$, and $\underline{\theta}_{D} \in \Omega_{D}$. Therefore, all of the adaptive laws have to be modified by using the parameters projection algorithm [4], [8], [12], such that the parameter vectors will remain inside the constraints. The modified adaptive laws are given as follows.

- Use the following adaptive law to adjust the parameter vector $\underline{\theta}_{f}$ :

$$
\underline{\dot{\theta}}_{f}= \begin{cases}-\gamma_{1} \underline{\xi}(\underline{\hat{x}}) B^{T} P \underline{\tilde{e}} & \text { if }\left(|\underline{\theta} f|<M_{f}\right) \\ & \text { or }\left(|\underline{\theta} f|=M_{f}\right. \\ & \text { and } \left.\underline{\tilde{e}}^{T} P B \underline{\xi}^{T}(\underline{\hat{x}}) \underline{\theta}_{f} \geq 0\right) \\ \operatorname{Proj}\left\{-\gamma_{1} \underline{\xi}(\underline{\hat{x}}) B^{T} \underline{\tilde{e}}\right\} & \text { if }\left(|\underline{\theta} f|=M_{f}\right. \\ & \text { and } \left.\underline{\tilde{e}}^{T} P B \underline{\xi}(\underline{\hat{x}}) \underline{\theta}_{f}<0\right)\end{cases}
$$

where the projection operator $\operatorname{Proj}\{*\}$ is defined as

$$
\begin{aligned}
\operatorname{Proj}\left\{-\gamma_{1} \underline{\xi}(\underline{\hat{x}}) B^{T} P \underline{\tilde{e}}\right\} & \\
& =-\gamma_{1} \underline{\xi}(\underline{\hat{x}}) B^{T} P \underline{\tilde{e}}+\gamma_{1} \underline{\tilde{e}}^{T} P B \frac{\underline{\theta}_{f} \underline{\theta}_{f}^{T} \underline{\xi}^{T}(\underline{\hat{x}})}{\left|\underline{\theta}_{f}\right|^{2}} .
\end{aligned}
$$

- Use the following adaptive law to adjust the parameter vector $\underline{\theta}_{g}$ :

$\square$ Whenever an element $q_{g i}^{l}$ in (16) of $\underline{\theta}_{g}=\varepsilon$, use

$$
\dot{q}_{g i}^{l}= \begin{cases}-\gamma_{2} \xi^{l}(\underline{\hat{x}}) B^{T} P \underline{\tilde{e}} u_{I} & \text { if } \underline{\underline{e}}^{T} P B \xi^{l}(\underline{\hat{x}}) u_{I}<0 \\ 0 & \text { if } \underline{\underline{e}}^{T} P B \xi^{l}(\underline{\hat{x}}) u_{I} \geq 0\end{cases}
$$

where $\xi^{l}(\underline{\hat{x}})$ is the $l$ th component of $\underline{\xi}^{T}(\underline{\hat{x}})$.

$$
\begin{aligned}
& \square \text { Otherwise, use } \\
& \dot{\underline{\theta}}_{g}= \begin{cases}-\gamma_{2} \underline{\xi}(\underline{\hat{x}}) B^{T} P \underline{\tilde{e}} u_{I} & \text { if }\left(|\underline{\theta} g|<M_{g}\right) \\
& \text { or }\left(|\underline{\theta} g|=M_{g}\right. \\
& \text { and } \underline{\tilde{e}}^{T} P B \underline{\xi}^{T}(\underline{\hat{x}}) \\
\operatorname{Proj}\left\{-\gamma_{2} \underline{\xi}(\underline{\hat{x}}) B^{T} P \underline{\tilde{e}} u_{I}\right\} & \underline{\underline{\theta}} u^{\left.u_{I} \geq 0\right)} \\
& \text { if }\left(|\underline{\theta} g|=M_{g}\right. \\
& \text { and } \underline{\tilde{e}}^{T} P B \underline{\xi}^{T}(\underline{\hat{x}}) \\
& \left.\underline{\theta} g^{u_{I}}<0\right)\end{cases}
\end{aligned}
$$

where the projection operator $\operatorname{Proj}\{*\}$ is defined as

$\operatorname{Proj}\left\{-\gamma_{2} \underline{\xi}(\underline{\hat{x}}) B^{T} P \underline{e} u_{I}\right\}$

$$
=-\gamma_{2} \underline{\xi}(\underline{\hat{x}}) B^{T} \underline{\tilde{e}} u_{I}+\gamma_{2} \underline{\tilde{e}}^{T} P B \frac{\underline{\theta}_{g} \underline{\theta}_{g}^{T} \underline{\xi}^{T}(\underline{\hat{x}}) u_{I}}{\left|\underline{\theta}_{g}\right|^{2}} .
$$

- Use the following adaptive law to adjust the parameter vector $\underline{\theta}_{D}$ :

$$
\underline{\dot{\theta}}_{D}= \begin{cases}\gamma_{3} \underline{\eta}(\underline{\hat{x}}) g(\underline{x}) B^{T} P \underline{\tilde{e}} & \text { if }\left(|\underline{\theta} D|<M_{D}\right) \\ & \text { or }\left(|\underline{\theta} D|=M_{D}\right. \\ & \text { and } \underline{\tilde{e}}^{T} P B g(\underline{x}) \underline{\eta}^{T}(\underline{\hat{x}}) \\ & \underline{\theta}{ } \geq 0) \\ \operatorname{Proj}\left\{\gamma_{3} \underline{\eta}(\underline{\hat{x}}) g(\underline{x}) B^{T} P \underline{\tilde{e}}\right\} & \text { if }\left(|\underline{\theta} D|=M_{D}\right. \\ & \text { and } \underline{\tilde{e}}^{T} P B g(\underline{x}) \underline{\eta}^{T}(\underline{\hat{x}}) \\ & \left.\underline{\theta}_{D}<0\right)\end{cases}
$$

where the projection operator $\operatorname{Proj}\{*\}$ is defined as

$$
\begin{aligned}
\operatorname{Proj} & \left\{\gamma_{3} \underline{\eta}(\underline{\hat{x}}) g(\underline{x}) B^{T} P \underline{\tilde{e}}\right\} \\
& =\gamma_{3} \tilde{\underline{e}}^{T} P B g(\underline{x}) \underline{\eta}(\underline{\hat{x}})-\gamma_{3} \underline{\tilde{e}}^{T} P B g(\underline{x}) \frac{\underline{\theta} D \underline{\theta}_{D}^{T} \underline{\eta}^{T}(\underline{\hat{x}})}{|\underline{\theta} D|^{2}} .
\end{aligned}
$$

Following the preceding consideration, we obtain the following theorem.

Theorem 1: Consider the plant (2) with control (24), where $u_{I}$ is given by (8) and $u_{S}$ is given by (22), and the fuzzy logic systems $\hat{f}, \hat{g}$, and $u_{D}$ are represented in (27) form. Let Assumption $I$ be true and the parameter vectors $\underline{\theta}_{f}, \underline{\theta}_{g}$, and $\underline{\theta}_{D}$ be adjusted by the adaptive laws (41)-(47). Then, the overall observer-based control scheme as shown in Fig. 2 guarantees the following properties:

1) $\left|\underline{\theta}_{f}\right| \leq M_{f},\left|\underline{\theta}_{g}\right| \leq M_{g},\left|\underline{\theta}_{D}\right| \leq M_{D}$, and all of the elements $q_{g i}^{l}$ in (16) of $\theta_{g} \geq \varepsilon$

$$
|\underline{\hat{x}}(t)| \leq\left[\left|\underline{y}_{r}\right|+\left(\frac{2 \overline{V_{\underline{\hat{e}}}}}{\lambda_{\hat{P} \min }}\right)^{1 / 2}\right]=M_{\underline{\underline{x}}}
$$

and

$$
\begin{aligned}
|u| \leq & \frac{\alpha}{\varepsilon}\left[M_{f \underline{\underline{\underline{x}}}}+\left|y_{r}^{(n)}\right|+\left|\underline{k}_{c}\right|\left(\frac{2 \bar{V}_{\underline{\underline{e}}}}{\lambda_{\hat{P} \min }}\right)^{1 / 2}\right] \\
& +(1-\alpha) M_{D \underline{\hat{x}}}+\frac{1}{g_{L}(\underline{x})}\left\{\left(1+\frac{g^{U}(\underline{x})+M_{g \underline{\underline{x}}}}{\varepsilon}\right)\right. \\
& \times\left[M_{f \underline{\hat{x}}}+\left|y_{r}^{(n)}\right|+\left|\underline{k}_{c}\right|\left(\frac{2 \bar{V}_{\underline{\underline{e}}}}{\lambda_{\hat{P} \min }}\right)^{1 / 2}\right] \\
& \left.+2 f^{U}(\underline{x})+g^{U}(\underline{x}) M_{D \underline{\underline{\hat{x}}}}+d_{m}\right\}
\end{aligned}
$$




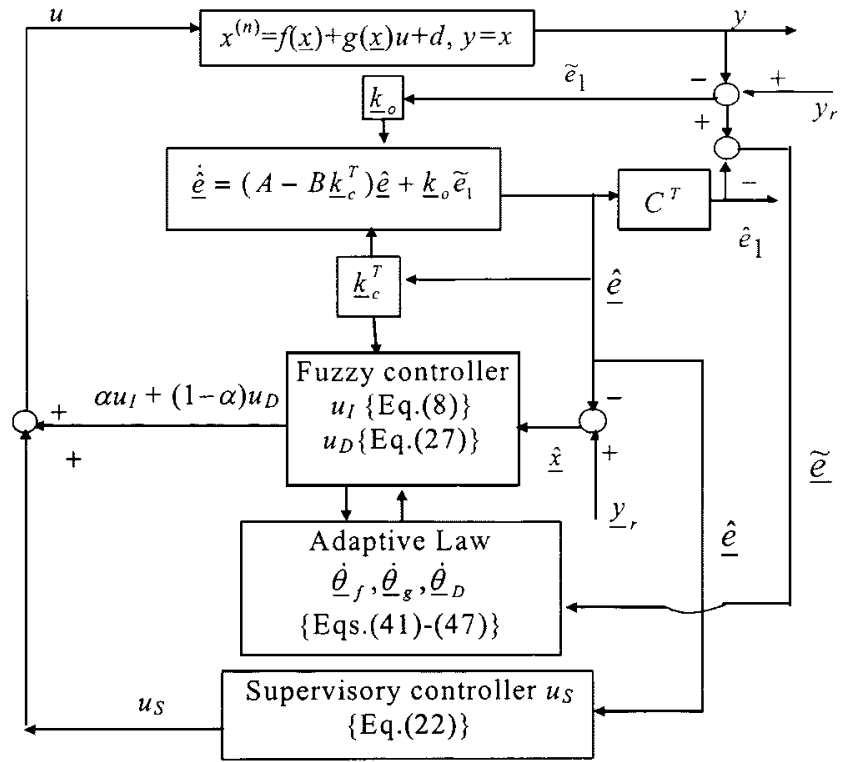

Fig. 2. Overall scheme of the observer-based hybrid direct/indirect adaptive FNN control.

for all $t \geq 0$, where $\lambda_{\hat{P} \text { min }}$ is the minimum eigenvalue of $\hat{P}, M_{f \underline{\hat{x}}}=M_{f}\left(1+M_{\underline{\hat{x}}}\right), M_{g \underline{\underline{\hat{x}}}}=M_{g}\left(1+M_{\underline{\hat{x}}}\right)$, and 2) $M_{D \underline{\hat{x}}}=M_{D}\left(1+M_{\underline{\hat{x}}}\right)$.

$$
\int_{0}^{t}|\underline{\tilde{e}}(\zeta)|^{2} d \zeta \leq a+b \int_{0}^{t}|\omega(\zeta)|^{2} d \zeta
$$

for all $t \geq 0$, where $a$ and $b$ are constants and $\omega$ is the minimum approximation error defined in (31).

3) If $\omega$ is squared integrable, i.e., $\int_{0}^{\infty}|\omega(t)|^{2} d t<\infty$, then $\lim _{t \rightarrow \infty}|\underline{\tilde{e}}(t)|=0$.

Proof:

I. i). To prove $\left|\underline{\theta}_{f}\right| \leq M_{f}$ :

A) Let $V_{f}=(1 / 2) \underline{\theta}_{f}^{T} \underline{\theta}_{f}$, if the first line of (41) is true, we have either $\left|\underline{\theta}_{f}\right| \leq M_{f}$ or, $\dot{V}_{f}=-\gamma_{1} \underline{\theta}_{f}^{T} \xi(\underline{\hat{x}}) B^{T} P \underline{\tilde{e}} \leq$ 0 for $\left|\underline{\theta}_{f}\right|=M_{f}$, i.e., we always have $\left|\underline{\theta}_{f}\right| \leq M_{f}$.

B) If the second line of (41), we have $\left|\underline{\theta}_{f}\right|=M_{f}$, and

$$
\begin{aligned}
\dot{V}_{f}=-\gamma_{1} \underline{\theta}_{f}^{T} & \underline{\xi}(\underline{x}) B^{T} P \underline{\tilde{e}} \\
& +\gamma_{1} \underline{\tilde{e}}^{T} P B \frac{\left|\underline{\theta}_{f}\right|^{2} \underline{\theta}_{f}^{T} \underline{\xi}(\underline{\hat{x}})}{\left|\underline{\theta}_{f}\right|^{2}}=0, \quad \text { i.e., }\left|\underline{\theta}_{f}\right| \leq M_{f} .
\end{aligned}
$$

Therefore, we prove that $\left|\underline{\theta}_{f}\right| \leq M_{f}, t \geq 0$.

ii) Use the similar method to show that $\left|\underline{\theta}_{g}\right| \leq M_{g},\left|\underline{\theta}_{D}\right| \leq$ $M_{D}, t \geq 0$.

From (43), we see that if $q_{g i}^{l}$ in (16) $=\varepsilon$, then $\dot{q}_{g i}^{l} \geq 0$; that is, we have $q_{g i}^{l} \geq \varepsilon$ for all elements $q_{g i}^{l}$ of $\underline{\theta}_{g}$.

iii) To prove (48).

In the above description, we prove that $V_{\underline{\underline{e}}} \leq \bar{V}_{\underline{\hat{e}}}$; therefore, $(1 / 2) \lambda_{\hat{P} \min }|\underline{\hat{e}}|^{2} \leq(1 / 2) \underline{\hat{e}}^{T} \hat{P} \underline{\hat{e}} \leq \bar{V}_{\underline{\hat{e}}} ;$ i.e.

$$
|\underline{\hat{e}}| \leq\left(\frac{2 \bar{V}_{\underline{\hat{e}}}}{\lambda_{\hat{P} \min }}\right)^{1 / 2} .
$$

Since $\underline{\hat{e}}=\underline{y}_{r}-\underline{\hat{x}}$, we have

$$
|\underline{\hat{x}}| \leq\left[\left|\underline{y}_{r}\right|+\left(\frac{2 \bar{V}_{\underline{\underline{\hat{e}}}}}{\lambda_{\hat{P} \min }}\right)^{1 / 2}\right]=M_{\underline{\hat{\hat{x}}}} .
$$

iv) To prove (49).

Since $\hat{f}\left(\underline{\hat{x}} \mid \underline{\theta}_{f}\right), \hat{g}\left(\underline{\hat{x}} \mid \underline{\theta}_{g}\right)$ and $u_{D}\left(\underline{\hat{x}} \mid \underline{\theta}_{D}\right)$ are weighted averages of the elements of $\underline{\theta}_{f}, \underline{\theta}_{g}$, and $\underline{\theta}_{D}$, respectively, we have

$$
\begin{aligned}
\left|\hat{f}\left(\underline{\hat{x}} \mid \underline{\theta}_{f}\right)\right| & \leq M_{f}\left(1+M_{\underline{\hat{x}}}\right)=M_{f \underline{\hat{x}}} \\
\left|\hat{g}\left(\underline{\hat{x}} \mid \underline{\theta}_{g}\right)\right| & \leq M_{g}\left(1+M_{\underline{\underline{x}}}\right)=M_{g \underline{\hat{x}}} \\
\left|u_{D}\left(\underline{\hat{x}} \mid \underline{\theta}_{D}\right)\right| & \leq M_{D}\left(1+M_{\underline{\hat{x}}}\right)=M_{D \underline{\hat{x}}}
\end{aligned}
$$

and $\left|\hat{g}\left(\underline{\hat{x}}^{\hat{\theta}} \underline{\underline{\theta}}_{g}\right)\right| \geq \varepsilon>0$ [since $q_{g i}^{l}$ in (16) $\geq \varepsilon$ ]. Therefore, from (8) we obtain

$$
\begin{aligned}
\left|u_{I}\right| & \leq \frac{1}{|\hat{g}(\underline{\hat{x}} \mid \underline{\theta} g)|}\left[\left|\hat{f}\left(\underline{\hat{x}} \mid \underline{\theta}_{f}\right)\right|+\left|y_{r}^{(n)}\right|+\left|\underline{k}_{c}\right| \cdot|\underline{\hat{e}}|\right] \\
& \leq \frac{1}{\left|\hat{g}\left(\underline{\hat{x}} \mid \underline{\theta}_{g}\right)\right|}\left[\left|\hat{f}\left(\underline{\hat{\hat{x}}} \mid \underline{\theta}_{f}\right)\right|+\left|y_{r}^{(n)}\right|+\left|\underline{k}_{c}\right|\left(\frac{2 \bar{V}_{\underline{\underline{e}}}}{\lambda_{\hat{P} \min }}\right)^{1 / 2}\right] \\
& \leq \frac{1}{\varepsilon}\left[M_{f \underline{\underline{\hat{x}}}}+\left|y_{r}^{(n)}\right|+\left|\underline{k}_{c}\right|\left(\frac{2 \bar{V}_{\underline{\underline{\hat{e}}}}}{\lambda_{\hat{P} \min }}\right)^{1 / 2}\right]
\end{aligned}
$$

According to (22) and (51)-(54), we manipulate them and have

$$
\begin{aligned}
\left|u_{S}\right| \leq & \frac{1}{g_{L}(\underline{x})}\left[\left|\hat{f}\left(\underline{\hat{x}} \mid \underline{\theta}_{f}\right)\right|+2 f^{U}(\underline{x})+\left|y_{r}^{(n)}\right|+\left|\underline{k}_{c}^{T} \underline{\hat{e}}\right|\right. \\
& \left.+g^{U}(\underline{x})\left(\left|u_{I}\right|+\left|u_{D}\right|\right)+\left|\hat{g}\left(\underline{\hat{x}} \mid \underline{\theta}_{g}\right) u_{I}\right|+d_{m}\right] \\
\leq & \frac{1}{g_{L}(\underline{x})}\left\{M_{f \underline{\hat{x}}}+\left|y_{r}^{(n)}\right|+\left|\underline{k}_{c}\right|\left(\frac{2 \bar{V}_{\underline{\underline{e}}}}{\lambda_{\hat{P} \min }}\right)^{1 / 2}\right. \\
& +\left(g^{U}(\underline{x})+M_{g \underline{\hat{x}}}\right) \\
& \times \frac{1}{\varepsilon}\left[M_{f \underline{\hat{x}}}+\left|y_{r}^{(n)}\right|+\left|\underline{k}_{c}\right|\left(\frac{2 \bar{V}_{\underline{\underline{e}}}}{\lambda_{\hat{P} \min }}\right)^{1 / 2}\right] \\
& \left.+2 f^{U}(\underline{x})+g^{U}(\underline{x}) M_{D \underline{\hat{x}}}+d_{m}\right\} \\
= & \frac{1}{g_{L}(\underline{x})}\left\{\left(1+\frac{\left.g^{U}(\underline{x})+M_{g \underline{\underline{x}}}\right)}{\varepsilon}\right.\right. \\
& \times\left[M_{f \underline{\hat{x}}}+\left|y_{r}^{(n)}\right|+\left|\underline{k}_{c}\right|\left(\frac{2 \bar{V}_{\underline{\underline{e}}}}{\lambda_{\hat{P} \min }}\right)^{1 / 2}\right] \\
& \left.+2 f^{U}(\underline{x})+g^{U}(\underline{x}) M_{D \underline{\underline{\hat{x}}}}+d_{m}\right\} .
\end{aligned}
$$

By combining (53)-(55) and substituting into (6), we can prove (49). 
II. From (36), and by using the modified adaptive laws in (41)-(47), we have

$$
\dot{V} \leq-\frac{1}{2} \underline{\tilde{e}}^{T} Q \underline{\tilde{e}}-g(\underline{x}) \underline{\tilde{e}}^{T} P B u_{S}+\underline{\tilde{e}}^{T} P B \omega .
$$

Since $g(\underline{x})>0$ and from (22), we have $g(\underline{x}) \underline{\tilde{e}}^{T} P B u_{S} \geq 0$. Hence, (56) can be simplified as

$$
\begin{aligned}
\dot{V} \leq & -\frac{1}{2} \underline{\tilde{e}}^{T} Q \underline{\tilde{e}}+\underline{\tilde{e}}^{T} P B \omega \\
\leq & -\frac{\lambda_{Q \min }-1}{2}|\underline{\tilde{e}}|^{2}-\frac{1}{2}\left(|\underline{\tilde{e}}|^{2}-2 \underline{\tilde{e}} P B \omega+|P B \omega|^{2}\right) \\
& +\frac{1}{2}|P B \omega|^{2} \\
\leq & -\frac{\lambda_{Q \min }-1}{2}|\underline{\tilde{e}}|^{2}+\left.\frac{1}{2} P B \omega\right|^{2}
\end{aligned}
$$

where $\lambda_{Q \text { min }}$ is the minimum eigenvalue of $Q$. By integrating both sides of (57) and assuming that $\lambda_{Q \text { min }}>1$ (since $Q$ is specified by the designer, we can choose such a $Q$ ), after some simple manipulations, we can obtain

$$
\begin{aligned}
\int_{0}^{t}|\underline{\tilde{e}}(\zeta)|^{2} d \zeta \leq & \frac{2}{\lambda_{Q \min }-1}(|V(0)+| V(t) \mid) \\
& +\frac{1}{\lambda_{Q \min }-1}|P B|^{2} \int_{0}^{t}|\omega(\zeta)|^{2} d \zeta .
\end{aligned}
$$

Defining $a=\left(2 /\left(\lambda_{Q \min }-1\right)\right)(|V(0)+| V(t) \mid)$ and $b=$ $\left(1 /\left(\lambda_{Q \min }-1\right)\right)|P B|^{2}$, we can prove (50) by substituting $a$ and $b$ into (58).

III. From (50), if $\omega \in L_{2}$, we have $\underline{\tilde{e}} \in L_{2}$. We have $\underline{\tilde{\tilde{e}}} \in$ $L_{\infty}$, because we have proven that all variables in the right-hand side of (33) are bounded. Using Barbalat's lemma [23] [if $\underline{\tilde{e}} \in$ $L_{2} \cap L_{\infty}$ and $\underline{\tilde{\tilde{e}}} \in L_{\infty}$, then $\lim _{t \rightarrow \infty}|\underline{\tilde{e}}(t)|=0$ ] we have $\lim _{t \rightarrow \infty}|\underline{\tilde{e}}(t)|=0$. This completes the proof.

Remark I: It is obvious that we need to know $g(\underline{x})$ beforehand in adaptive law (39), i.e., in the above theorem the adaptive FNN control works under those nonlinear systems of which $g(\underline{x})$ is well known. If the dynamics $g(\underline{x})$ can be split into a well-known nominal part $g_{0}(\underline{x})$, plus an uncertain part $g_{d}(\underline{x})$, then $g_{d}(\underline{x}) u$ can be considered as a part of the external disturbance. In the meantime, it can be attenuated by the proposed methodology.

To summarize the above analysis, the design algorithm for observer-based hybrid direct/indirect adaptive FNN control is proposed as follows.

Step 1) Specify the feedback and observer gain vector $\underline{k}_{c}$ and $\underline{k}_{o}$, such that the characteristic matrices $A-$ $B \underline{k}_{c}^{T}$ and $A-\underline{k}_{o} C^{T}$ are strictly Hurwitz matrices, respectively.

Step 2) Specify a positive definite $n \times n$ matrix $Q$ and solve the Lyapunov equation (12) to obtain a positive definite symmetric $n \times n$ matrix $P$.

Step 3) Solve the state error equation (10) to obtain estimate state vector $\underline{\hat{x}}=y_{r}-\underline{\hat{e}}$.

Step 4) Specify the parameters $M_{f}, M_{g}, M_{D}, M_{\underline{\hat{x}}}, \gamma_{1}, \gamma_{2}$, $\gamma_{3}, \varepsilon$, and $\bar{V}$ based on the practical constraints. Although $\bar{V}$ is any given constant, we let $\bar{V}$ be the same as $\bar{V}_{\underline{\underline{e}}}$ (described at the end of Section II), which

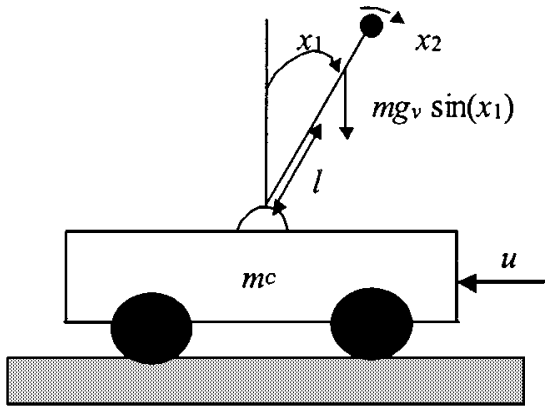

Fig. 3. Inverted pendulum system.

can be determined from $M_{\underline{\underline{\hat{x}}}},\left|y_{r}\right|$ and $\lambda_{\hat{P} \min }$ of $\hat{P}$ in (48). This is to match the magnitude scale of the system so that the designer is free from supplying $\bar{V}$ at random to the system.

Step 5) Define the membership function $\mu_{F^{l}}(\underline{\hat{x}})$ for $i=$ $1,2, \ldots, M$ and compute the fuzzy basis functions $\underline{\xi}(\hat{\underline{x}})$. Then, fuzzy logic control systems are constructed as

$$
\hat{f}\left(\underline{\hat{x}} \mid \underline{\theta}_{f}\right)=\underline{\xi}^{T}(\underline{\hat{x}}) \underline{\theta}_{f}, \quad \hat{g}\left(\underline{\hat{x}} \mid \underline{\theta}_{g}\right)=\underline{\xi}^{T}(\underline{\hat{x}}) \underline{\theta}_{g} .
$$

Similarly, define the other membership functions and compute $\eta(\underline{\hat{x}})$. Then, fuzzy logic control system is constructed as

$$
u_{D}\left(\underline{\hat{x}} \mid \underline{\theta}_{D}\right)=\underline{\eta}^{T}(\underline{\hat{x}}) \underline{\theta}_{D} .
$$

Step 6) Obtain the control and apply it to the plant, then compute the adaptive laws (41)-(47) to adjust the parameter vectors $\underline{\theta}_{f}, \underline{\theta}_{g}$, and $\underline{\theta}_{D}$. Following Remark $I$, we let the unknown $g(\underline{x})$ be $g_{0}(\underline{x})$ in (46) and (47).

\section{EXAMPLES}

In this section, we will apply our observer-based hybrid direct/indirect adaptive FNN controller to control inverted pendulum and Chua's chaotic circuit to track a sine-wave trajectory.

Example 1: Consider the inverted pendulum system shown in Fig. 3. Let $x_{1}=\theta$ be the angle of the pendulum with respect to the vertical line.

The dynamic equations of the inverted pendulum system [4], [8], [12], [23] are

$$
\begin{aligned}
{\left[\begin{array}{c}
\dot{x}_{1} \\
\dot{x}_{2}
\end{array}\right] } & =\left[\begin{array}{ll}
0 & 1 \\
0 & 0
\end{array}\right]\left[\begin{array}{l}
x_{1} \\
x_{2}
\end{array}\right]+\left[\begin{array}{l}
0 \\
1
\end{array}\right](f+g u+d) \\
y & =\left[\begin{array}{ll}
1 & 0
\end{array}\right]\left[\begin{array}{l}
x_{1} \\
x_{2}
\end{array}\right]
\end{aligned}
$$

where

$$
f=\frac{g_{v} \sin x_{1}-\frac{m l x_{2}^{2} \cos x_{1} \sin x_{1}}{m_{c}+m}}{l\left(\frac{4}{3}-\frac{m \cos ^{2} x_{1}}{m_{c}+m}\right)} ; \quad g=\frac{\frac{\cos x_{1}}{m_{c}+m}}{l\left(\frac{4}{3}-\frac{m \cos ^{2} x_{1}}{m_{c}+m}\right)}
$$

and $g_{v}=9.8 \mathrm{~m} / \mathrm{s}^{2}$ is the acceleration due to gravity; $m_{c}$ is the mass of the cart; $l$ is the half-length of the pole; $m$ is the mass of the pole; and $u$ is the control input. In this example, we assume 
that $m_{c}=1 \mathrm{~kg}, m=0.1 \mathrm{~kg}$, and $l=0.5 \mathrm{~m}$. It is obvious that $0<g<\infty$ so that Assumption I in Section IV is satisfied. This is due to $\cos x_{1} /\left(m_{c}+m\right)>0$ and $m \cos ^{2} x_{1} /\left(m_{c}+m\right)<$ 1. We also have to determine the bounds $f^{U}, g^{U}$, and $g_{L}$ as follows:

$$
\begin{aligned}
\left|f\left(x_{1}, x_{2}\right)\right| & \leq \frac{9.8+\left(0.025 x_{2}^{2}\right)}{(2 / 3)-(0.05 / 1.1)}=15.78+0.0366 x_{2}^{2} \\
& \approx 15.78+0.0366 \hat{x}_{2}^{2}=f^{U}\left(\hat{x}_{1}, \hat{x}_{2}\right) \\
\left|g\left(x_{1}, x_{2}\right)\right| & \leq 1.46=g^{U}\left(x_{1}, x_{2}\right) \approx g^{U}\left(\hat{x}_{1}, \hat{x}_{2}\right)
\end{aligned}
$$

and if we require that $\left|x_{1}\right| \leq \pi / 6$, then

$$
\left|g\left(x_{1}, x_{2}\right)\right| \geq 1.12=g_{L}\left(x_{1}, x_{2}\right) \approx g_{L}\left(\hat{x}_{1}, \hat{x}_{2}\right) .
$$

The control object is to control the state $x_{1}$ of the system to track the reference trajectory $y_{r}(t)=(\pi / 30) \sin (t)$ if only the system output $y$ is measurable. Also, the external disturbance $d$ is assumed to be a square-wave with amplitude \pm 0.1 , period $2 \pi$, and the parameters are chosen as $\gamma_{1} \approx 45.1, \gamma_{2} \approx 11.2$, $\gamma_{3} \approx 15.1$, and step size $h=0.00285$. The choices of $\gamma \mathrm{s}$ and $h$ are to improve the convergence rate of the closed-loop system controlled by our proposed controller.

According to the design procedure, the design is given in the following steps.

Step 1) The observer and feedback gain vectors are chosen as $\underline{k}_{o}^{T}=\left[\begin{array}{ll}89 & 184\end{array}\right]$ and $\underline{k}_{c}^{T}=\left[\begin{array}{ll}4 & 4\end{array}\right]$, respectively.

Step 2) We select $Q$ in (12) as $\left[\begin{array}{lll}10 & 13 \\ 13 & 28\end{array}\right]$, then after solving (12), the positive definite symmetric $2 \times 2$ matrix $P$ in (12) is $\left[\begin{array}{rr}29 & -14 \\ -14 & 7\end{array}\right]$. The minimum eigenvalue of $Q$, i.e., $\lambda_{Q \min }$ is 3.19 , which satisfies the transition from (56) to (57).

Step 3) Solve (10) to obtain $\underline{\hat{x}}$.

Step 4) We select $M_{f}=20, M_{g}=20, M_{d}=20, M_{\hat{x}}=$ $\pi / 6$, and $\varepsilon=0.3$, and $\hat{Q}$ in (14) is chosen as $\left[\begin{array}{c}40^{-} \\ 25 \\ 25\end{array}\right]$ and $\hat{A}=\left[\begin{array}{rr}0 & 1 \\ -4 & -4\end{array}\right]$ in (14). Therefore, the positive definite symmetric $2 \times 2$ matrix $\hat{P}$ in (14) can be solved as $\left[\begin{array}{cc}15 & 5 \\ 5 & 5\end{array}\right]$. The minimum eigenvalue of value $\hat{P}$, i.e., the $\lambda_{\hat{\Gamma} \min }$ in (48), is 2.93. Therefore, we can have $\bar{V}_{\hat{e}}$ from (48) as 0.257 .

Step 5) The following membership functions for $\hat{x}_{i}, i=$ 1,2 are selected as:

$$
\begin{aligned}
& \mu_{F_{1}^{i}}\left(\hat{x}_{i}\right)=\exp \left[-\left(\frac{\hat{x}_{i}+\pi / 6}{\pi / 24}\right)^{2}\right] \\
& \mu_{F_{2}^{i}}\left(\hat{x}_{i}\right)=\exp \left[-\left(\frac{\hat{x}_{i}+\pi / 12}{\pi / 24}\right)^{2}\right] \\
& \mu_{F_{3}^{i}}\left(\hat{x}_{i}\right)=\exp \left[-\left(\frac{\hat{x}_{i}}{\pi / 24}\right)^{2}\right] \\
& \mu_{F_{4}^{i}}\left(\hat{x}_{i}\right)=\exp \left[-\left(\frac{\hat{x}_{i}-\pi / 12}{\pi / 24}\right)^{2}\right] \\
& \mu_{F_{5}^{i}}\left(\hat{x}_{i}\right)=\exp \left[-\left(\frac{\hat{x}_{i}-\pi / 6}{\pi / 24}\right)^{2}\right] .
\end{aligned}
$$

\begin{tabular}{|c|c|c|c|}
\hline Cases & Initial states & Cases & Initial states \\
\hline Case1 & $\begin{array}{c}x(0)=\left[\begin{array}{ll}0.1 & 0\end{array}\right]^{T} \\
\hat{x}(0)=\left[\begin{array}{ll}-0.15 & 0\end{array}\right]^{T}\end{array}$ & Case 3 & $\begin{array}{l}x(0)=\left[\begin{array}{ll}-0.2 & 0\end{array}\right]^{T} \\
\hat{x}(0)=\left[\begin{array}{ll}0.15 & 0\end{array}\right]^{T}\end{array}$ \\
\hline Case 2 & $\begin{array}{c}x(0)=\left[\begin{array}{ll}0.25 & 0.25\end{array}\right]^{T} \\
\hat{x}(0)=\left[\begin{array}{ll}0.1 & 0.1\end{array}\right]^{T}\end{array}$ & Case4 & $\begin{array}{c}x(0)=\left[\begin{array}{ll}-0.25 & -0.25\end{array}\right]^{T} \\
\hat{x}(0)=\left[\begin{array}{ll}0.25 & 0.25\end{array}\right]^{T}\end{array}$ \\
\hline
\end{tabular}

To cover whole cases, we apply 25 fuzzy rules. For simplification, we let $\underline{\xi}(\underline{\hat{x}})=\underline{\eta}(\underline{\hat{x}})$.

Hence, $u_{I}$ and $u_{D}$ are constructed.
TABLE I

FOUR CASES OF THE INITIAL STATES

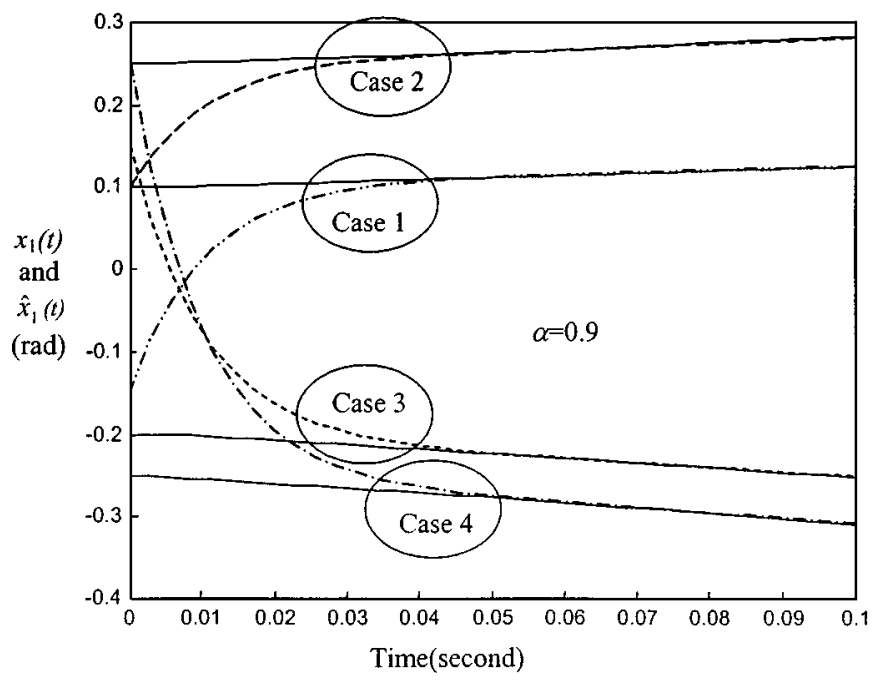

Fig. 4. Trajectories of the states $x_{1}$ (solid line) and $\hat{x}_{1}$ (dashed line) of four cases.

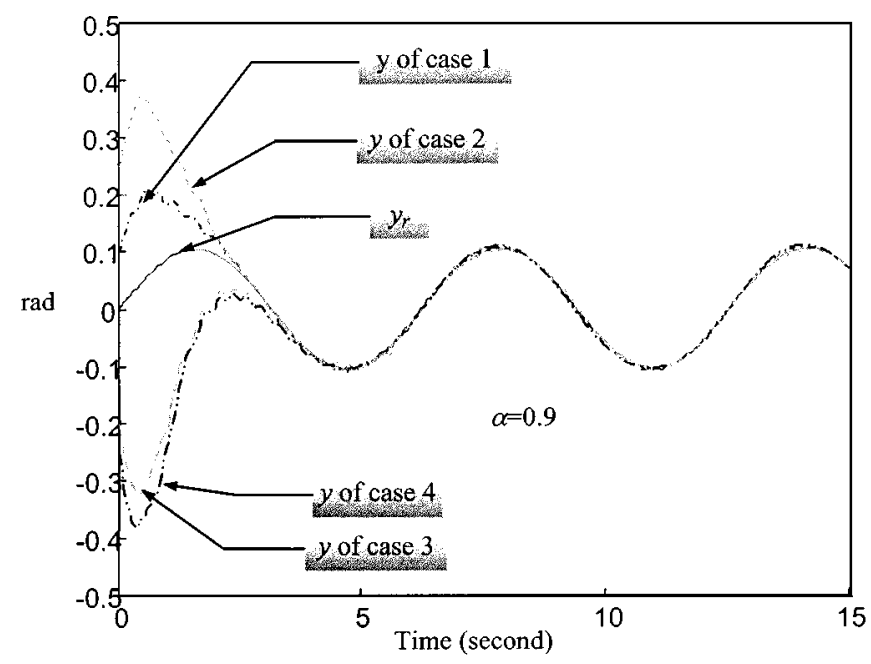

Fig. 5. Output trajectories $y$ of four cases and reference $y_{r}$ with $\alpha=0.9$.

Step 6) Compute the adaptive laws (41)-(47). From (60) and (61), we can let $g_{0}\left(x_{1}, x_{2}\right)=1$ to replace the unknown $g\left(x_{1}, x_{2}\right)$ in (46) and (47). This has been explained in Remark $I$.

According to the initial states, four cases are simulated, as shown in Table I.

Fig. 4 shows the trajectories of the states $x_{1}$ and $\hat{x}_{1}$ of four cases if $\alpha=0.9$ is chosen and it shows that the estimation state $\hat{x}_{1}$ takes very short time to catch up to the system state $x_{1}$.

The tracking performances of four cases are also very good, as shown in Fig. 5, in which $y_{r}$ is the reference trajectory and $y$ is the system output trajectory. This result is better, as shown in [4] and [12]. 


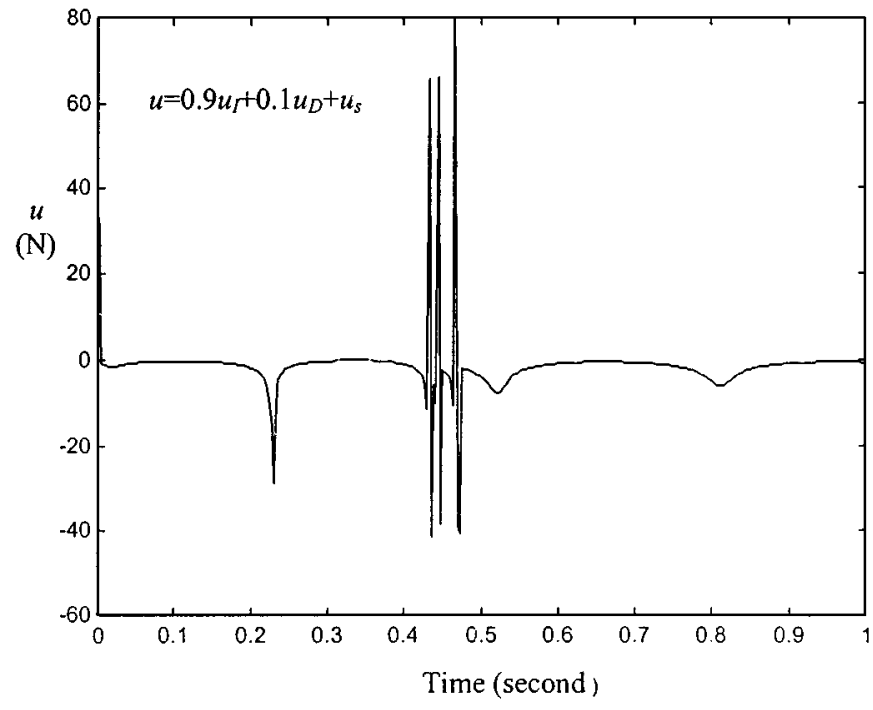

Fig. 6. Trajectory of the control input (include supervisory control) of Case 1 with $\alpha=0.9$ (time $=0 \sim 1 \mathrm{~s}$ ).

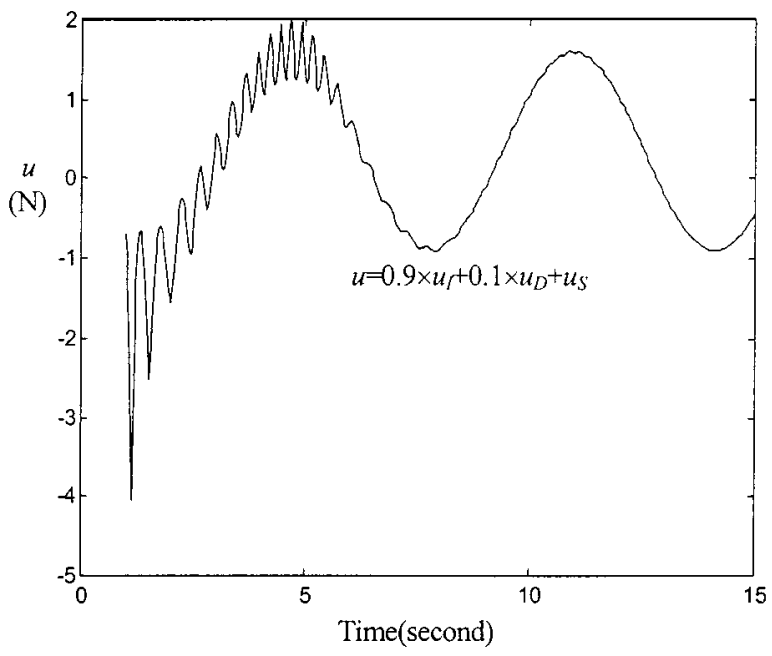

Fig. 7. Trajectory of the control input (include supervisory control) of Case 1 with $\alpha=0.9$ (time $=1 \sim 15 \mathrm{~s}$ ).

We show the control input $u=\alpha u_{I}+(1-\alpha) u_{D}+u_{S}$ of Case 1 with $\alpha=0.9$ in Figs. 6 and 7.

Fig. 8 shows the supervisory control $u_{S}$, and one can obviously see that it is activated in four periods: [0, 0.0057], [0.4361, 0.4389], [0.4475, 0.4503], and [0.4703, 0.4760]. After time = $0.4760 \mathrm{~s}$, the FNN controller can stabilize the system and the supervisory controller will never be activated again. The spikes in Figs. 6 and 8 are caused by the fact that $u_{S}$ must maintain a larger initial value to stabilize the system when the system tends to be unstable. Therefore, the adaptive controller can perform successful control and the desired performance can be achieved.

Applying the different weighting factor $\alpha$, the tracking error performance of Case 1 is shown in Fig. 9.

Example 2: The typical Chua's chaotic circuit in Fig. 10 consists of one linear resistor $(R)$, two capacitors $(C 1, C 2)$, one inductor, and one piecewise-linear resistor $(\lambda)$ [16], [24]. It has been shown to own very rich nonlinear dynamics such as chaos and bifurcations.

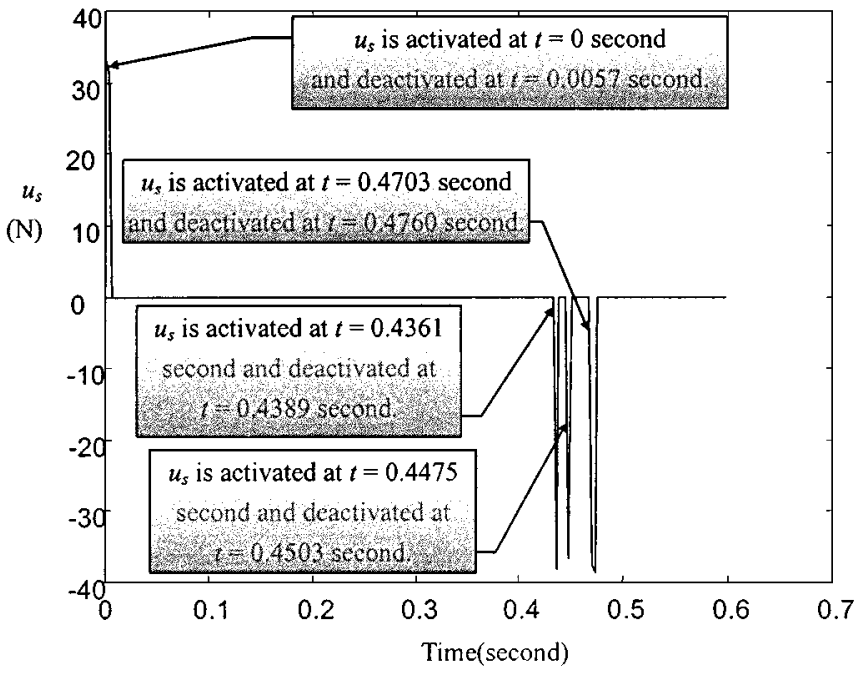

Fig. 8. Trajectory of the supervisory control $u_{S}$ of Case 1 with $\alpha=0.9$ (time $=0 \sim 0.6 \mathrm{~s})$.

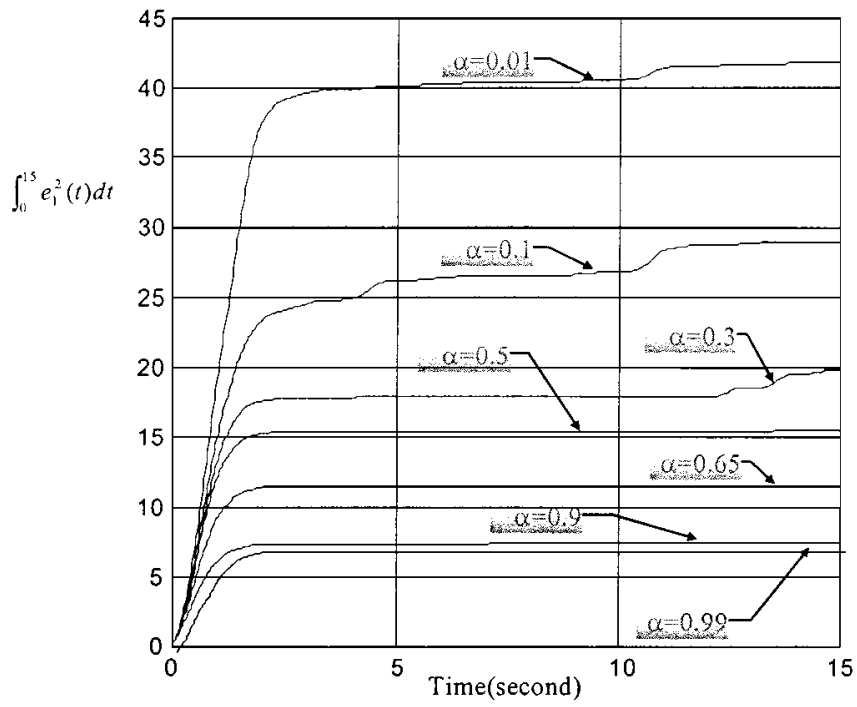

Fig. 9. Tracking performance $\int_{0}^{15} e_{1}^{2}(t) d t$ for Case 1 with different $\alpha$.

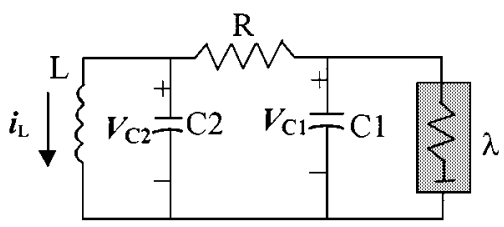

Fig. 10. Chua's chaotic circuit.

The dynamic equations of Chua's chaotic circuit are written as

$$
\begin{aligned}
\dot{V}_{C 1} & =\frac{1}{C 1}\left(\frac{1}{R}\left(V_{C 2}-V_{C 1}\right)-\lambda\left(V_{C 1}\right)\right) \\
\dot{V}_{C 2} & =\frac{1}{C 2}\left(\frac{1}{R}\left(V_{C 1}-V_{C 2}\right)+i_{L}\right) \\
\dot{i}_{L} & =\frac{1}{L}\left(-V_{C 1}-R_{0} i_{L}\right)
\end{aligned}
$$

where voltages $V_{C 1}$ and $V_{C 2}$ and current $i_{L}$ are state variables; $R_{0}$ is a constant; and $\lambda$ denotes the nonlinear resistor, which is a function of the voltage across the two terminals of $C 1$. Here, 


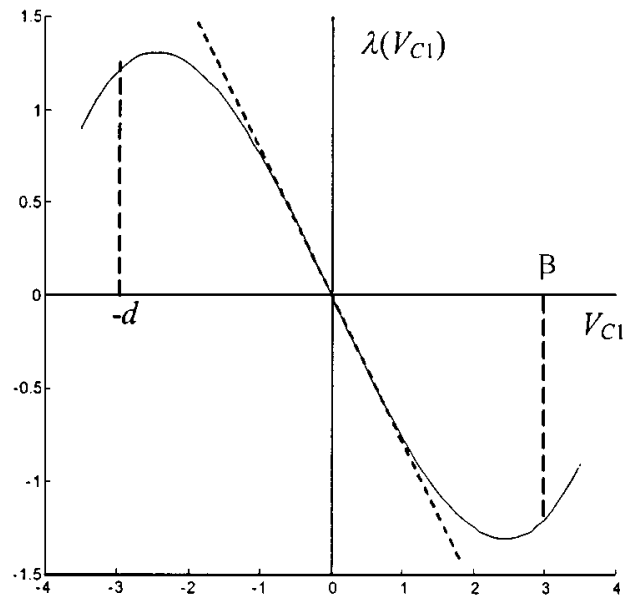

Fig. 11. Nonlinear resistor characteristics.

we define $\lambda$ as a cubic function as in (63), and its diagram is shown in Fig. 11 [24]

$$
\lambda\left(V_{C 1}\right)=a V_{C 1}+c V_{C 1}^{3} \quad(a<0, c>0) .
$$

The system can be rewritten as

$$
\dot{Z}(t)=G Z(t)+H \lambda
$$

where $Z=\left[\begin{array}{lll}z_{1} & z_{2} & z_{3}\end{array}\right]^{T}=\left[\begin{array}{lll}V_{C 1} & V_{C 2} & i_{L}\end{array}\right]^{T}$

$$
G=\left[\begin{array}{ccc}
-\frac{1}{C_{1} R} & \frac{1}{C_{1} R} & 0 \\
\frac{1}{C_{2} R} & -\frac{1}{C_{2} R} & \frac{1}{C_{2}} \\
0 & -\frac{1}{L} & -\frac{R_{0}}{L}
\end{array}\right] \text { and } H=\left[\begin{array}{c}
-\frac{1}{C_{1}} \\
0 \\
0
\end{array}\right] .
$$

The above state space equations are not in the standard canonical form defined in (3). Therefore, we need to perform a linear transformation to transform them into the form of (3). Let us define $Z(t)=T Z^{*}(t)$ or $Z^{*}(t)=T^{-1} Z(t)$ where $T$ is a transformation matrix. Using the transformation in [25] and [26], the transformed system can be obtained as

$$
\dot{Z}^{*}(t)=T^{-1} G T Z^{*}(t)+T^{-1} H \lambda=G^{*} Z^{*}(t)+H^{*} \lambda
$$

where, as shown in the equation at the bottom of the page, $G^{*}=$ $T^{-1} G T, H^{*}=T^{-1} H$.
Choose the parameters as follows:

$$
\begin{array}{lll}
R=\frac{10}{7}, & R_{0}=0, \quad C_{1}=1, \quad C_{2}=\frac{19}{2} \\
L=\frac{19}{14}, & a=-\frac{4}{5}, \quad c=\frac{2}{45}, \quad \beta=3 .
\end{array}
$$

Therefore, after computation, we get the transformed system as follows:

$$
\begin{aligned}
\dot{z}_{1}^{*}= & z_{2}^{*} ; \quad \dot{z}_{2}^{*}=z_{3}^{*} \\
\dot{z}_{3}^{*}= & \frac{14}{1805} z_{1}^{*}-\frac{168}{9025} z_{2}^{*}+\frac{1}{38} z_{3}^{*} \\
& -\frac{2}{45}\left(\frac{28}{361} z_{1}^{*}+\frac{7}{95} z_{2}^{*}+z_{3}^{*}\right)^{3} .
\end{aligned}
$$

For comparison, the simulation results of Chua's chaotic circuit and its transformed system are shown in Fig. 12.

We will design the hybrid FNN adaptive controller to dominate the transformed system to track a reference signal. For convenience, we let $\underline{x}$ replace $\underline{z}^{*}$ in the above transformed system. Therefore, the closed-loop configuration of (66) can be represented by

$$
\left[\begin{array}{l}
\dot{x}_{1} \\
\dot{x}_{2} \\
\dot{x}_{3}
\end{array}\right]=\left[\begin{array}{lll}
0 & 1 & 0 \\
0 & 0 & 1 \\
0 & 0 & 0
\end{array}\right]\left[\begin{array}{l}
x_{1} \\
x_{2} \\
x_{3}
\end{array}\right]+\left[\begin{array}{l}
0 \\
0 \\
1
\end{array}\right](f+g u+d)
$$

and

$$
y=\left[\begin{array}{lll}
1 & 0 & 0
\end{array}\right]\left[\begin{array}{l}
x_{1} \\
x_{2} \\
x_{3}
\end{array}\right]
$$

where

$$
\begin{aligned}
f=\frac{14}{1805} x_{1}-\frac{168}{9025} x_{2}+ & \frac{1}{38} x_{3}-\frac{2}{45} \\
& \times\left(\frac{28}{361} x_{1}+\frac{7}{95} x_{2}+x_{3}\right)^{3}, \quad g=1
\end{aligned}
$$

and $d$ is the external disturbance. Although the $f$ above is well defined since the Chua's circuit is well specified, we do not apply it in the adaptive law. However, we can indeed use it to estimate the upper bound of $f$, which is required in our design

and

$$
T=\left[\begin{array}{ccc}
-\frac{R+R_{0}}{C_{1} C_{2} R L} & -\frac{R R_{0} C_{2}+L}{C_{1} C_{2} R L} & -\frac{1}{C_{1}} \\
-\frac{R_{0}}{C_{1} C_{2} R L} & -\frac{1}{C_{1} C_{2} R} & 0 \\
\frac{1}{C_{1} C_{2} R L} & 0 & 0
\end{array}\right]
$$

$$
G^{*}=\left[\begin{array}{ccc}
0 & 1 & 0 \\
0 & 0 & 1 \\
-\frac{1}{C_{1} C_{2} R L} & -\frac{C_{1} R+C_{2} R_{0}+C_{1} R_{0}}{C_{1} C_{2} R L} & -\frac{C_{1} C_{2} R R_{0}+C_{2} L+C_{1} L}{C_{1} C_{2} R L}
\end{array}\right]
$$



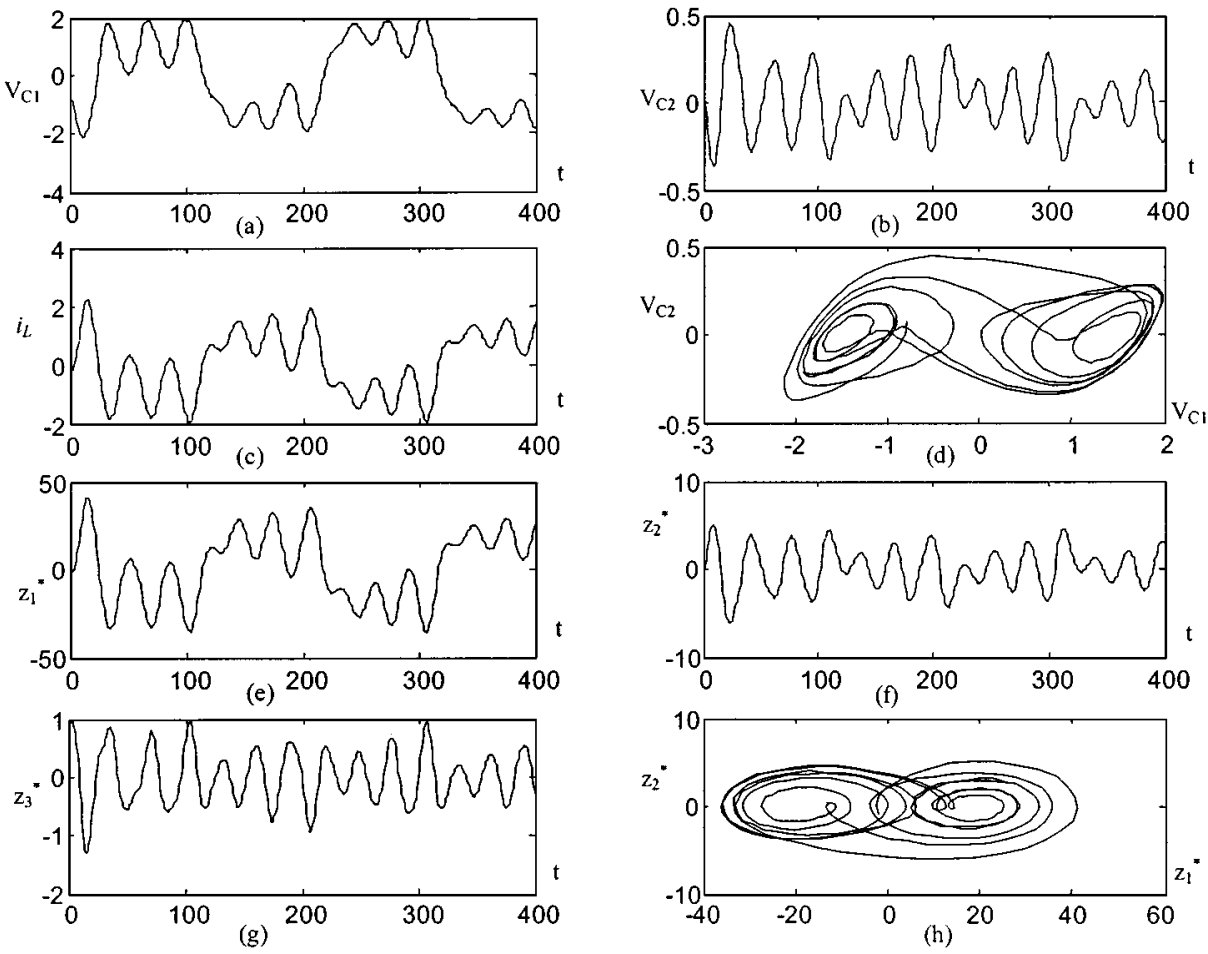

Fig. 12. (a) $V_{C 1}$ of Chua's circuit. (b) $V_{C 2}$ of Chua's circuit. (c) $i_{L}$ of Chua's circuit. (d) Phase-plane trajectory of Chua's circuit. (e) $z_{1}^{*}$ of transformed system. (f) $z_{2}^{*}$ of transformed system. (g) $z_{3}^{*}$ of transformed system. (h) Phase-plane trajectory of transformed system.

procedure. The bounds $f^{U}, g^{U}$, and $g_{L}$ can be estimated as follows:

$$
\begin{aligned}
\mid f( & \left.x_{1}, x_{2}, x_{3}\right) \mid \\
\leq & 14 / 1805 \cdot\left|x_{1}\right|+168 / 9025 \cdot\left|x_{2}\right|+1 / 38 \cdot\left|x_{3}\right| \\
& +2 / 45\left(28 / 361\left|x_{1}\right|+7 / 95\left|x_{2}\right|+\left|x_{3}\right|\right)^{3} \\
\leq & 14 / 1805 \times 50+168 / 9025 \times 10+1 / 38 \times 2 \\
& +2 / 45(28 / 361 \times 50+7 / 95 \times 10+2)^{3} \\
\approx & 13.5 \approx 14 / 1805 \cdot\left|\hat{x}_{1}\right|+168 / 9025 \cdot\left|\hat{x}_{2}\right|+1 / 38 \cdot\left|\hat{x}_{3}\right| \\
& +2 / 45\left(28 / 361\left|\hat{x}_{1}\right|+7 / 95\left|\hat{x}_{2}\right|+\left|\hat{x}_{3}\right|\right)^{3} \\
= & f^{U}\left(\hat{x}_{1}, \hat{x}_{2}, \hat{x}_{3}\right) .
\end{aligned}
$$

The above estimation comes from several simulation runs of the uncontrolled and transformed Chua's circuit in (66). Since $g=1$, we let

$$
g_{0}=1, \quad g^{U}\left(x_{1}, x_{2}, x_{3}\right) \approx g^{U}\left(\hat{x}_{1}, \hat{x}_{2}, \hat{x}_{3}\right)=1
$$
and

$$
g_{L}\left(x_{1}, x_{2}, x_{3}\right) \approx g_{L}\left(\hat{x}_{1}, \hat{x}_{2}, \hat{x}_{3}\right)=1
$$

The control object is to control the state $x_{1}$ of the system to track the reference trajectory $y_{r}(t)=1.5 \sin (t)$ if only the system output $y$ is measurable. Therefore, in the phase plane, this reference trajectory is a circle with radius 1.5: $y_{r}^{2}+\dot{y}_{r}^{2}=1.5$. Also the external disturbance $d$ is assumed to be a square-wave with amplitude \pm 0.5 , period $2 \pi$ and the parameters are chosen as $\gamma_{1} \approx 0.07771, \gamma_{2} \approx 0.00832$, $\gamma_{3} \approx 0.03808$, and step size $h=0.00255$. The choices of $\gamma \mathrm{s}$ and $h$ are to improve the convergence rate of the closed-loop system controlled by our proposed controller.

According to the design procedure, the design is given in the following steps.

Step 1) The observer and feedback gain vectors are chosen as $\underline{k}_{o}^{T}=\left[\begin{array}{lll}5 & 237 & 3\end{array}\right]$ and $\underline{k}_{c}^{T}=\left[\begin{array}{lll}12 & 13 & 3\end{array}\right]$, respectively.

Step 2) We select $Q$ in (12) as $6 \times I_{3 \times 3}$, then after solving (12), the positive definite symmetric $3 \times 3$ matrix $P$ in $(12)$ is

$$
\left[\begin{array}{ccc}
143.2233 & -3 & -0.7056 \\
-3 & 0.7055 & -3 \\
-0.7056 & -3 & 237.1759
\end{array}\right]
$$

The minimum eigenvalue of $Q$, i.e., $\lambda_{Q \min }$ is 6 , which satisfies the transition from (56) to (57).

Step 3) Solve (10) to obtain $\underline{\hat{x}}$.

Step 4) We select $M_{f}=50, M_{g}=50, M_{d}=50, M_{\underline{\hat{x}}}=$ 2.5 , and $\varepsilon=0.3$, and $\hat{Q}$ in (14) is chosen as $10 \times \bar{I}_{3 \times 3}$ and $\hat{A}$ in (14) is computed. Therefore, the positive definite symmetric $3 \times 3 \hat{P}$ in (14) can be solved as

$$
\left[\begin{array}{rrr}
40.4166 & 32.9166 & 0.4166 \\
32.9166 & 42.6388 & 2.9166 \\
0.4166 & 2.9166 & 2.6388
\end{array}\right] \text {. }
$$

The minimum eigenvalue of value $\hat{P}$, i.e., the $\lambda_{\hat{P} \min }$ in (48) is 2.1. Therefore, we can have $\bar{V}_{\hat{\underline{e}}}$ from (48) as 1.05 . 


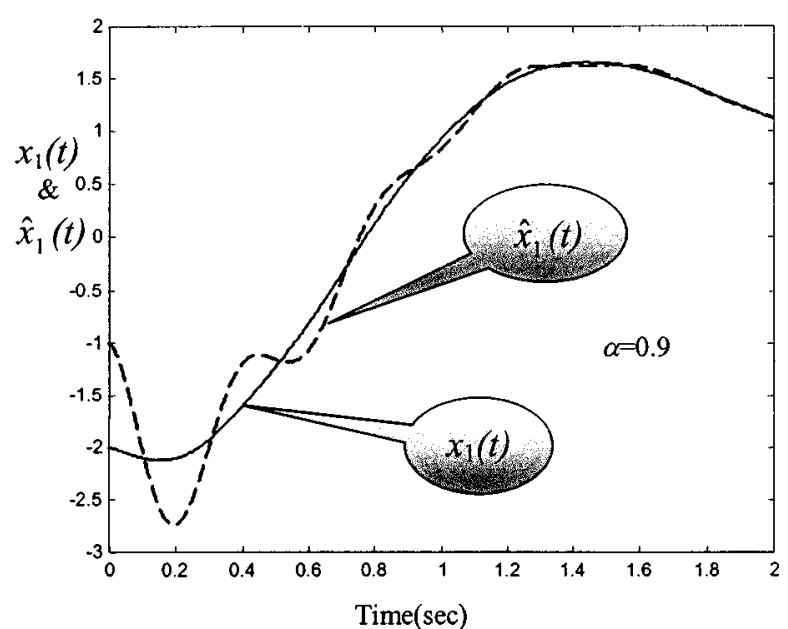

Fig. 13. Trajectories of the states $x_{1}$ (solid line) and $\hat{x}_{1}$ (dashed line).

Step 5) The following membership functions are selected as:

$$
\begin{aligned}
& \mu_{F_{1}^{i}}\left(\hat{x}_{i}\right)=1 /\left\{1+\exp \left[5\left(\hat{x}_{i}+\tau \cdot \zeta_{1}\right)\right]\right\}, \\
& \mu_{F_{2}^{i}}\left(\hat{x}_{i}\right)=\exp \left[-\left(\hat{x}_{i}+\tau \cdot \zeta_{2}\right)^{2}\right] \\
& \mu_{F_{3}^{i}}\left(\hat{x}_{i}\right)=\exp \left[-\left(\hat{x}_{i}+\tau\right)^{2}\right], \\
& \mu_{F_{4}^{i}}\left(\hat{x}_{i}\right)=\exp \left[-\left(\hat{x}_{i}-\tau\right)^{2}\right] \\
& \mu_{F_{5}^{i}}\left(\hat{x}_{i}\right)=\exp \left[-\left(\hat{x}_{i}-\tau \cdot \zeta_{2}\right)^{2}\right], \\
& \mu_{F_{6}^{i}}\left(\hat{x}_{i}\right)=1 /\left\{1+\exp \left[-5\left(\hat{x}_{i}-\tau \cdot \zeta_{1}\right)\right]\right\} .
\end{aligned}
$$

Let $\tau=1, \zeta_{1}=3.7, \zeta_{2}=3.3$, for $\hat{x}_{i}, i=1,2$. Set $\tau=3.8, \zeta_{1}=3.3, \zeta_{2}=2.6$, for $\hat{x}_{3}$.

To cover whole cases, we apply 216 fuzzy rules. For simplification, we let $\underline{\xi}(\underline{\hat{x}})=\underline{\eta}(\underline{\hat{x}})$. Hence, $u_{I}$ and $u_{D}$ are constructed.

Step 6) Compute the adaptive laws (41)-(47). From (69) and (70), it is obvious that we can let $g_{0}(\underline{x})=1$ to replace the unknown $g(\underline{x})$ in (46) and (47). This has been explained in the Remark $I$.

Fig. 13 shows the trajectories of the states $x_{1}$ and $\hat{x}_{1}$ if $\alpha=$ 0.9 is chosen and it shows that the estimation state $\hat{x}_{1}$ takes less than $1.4 \mathrm{~s}$ to catch up to the system state $x_{1}$.

Fig. 14(a)-(c) shows the responses of the transformed Chua's circuit. Fig. 14(d)-(f) shows the responses of the original Chua's circuit by restoring the transformed system to its original states.

Fig. 15 shows the phase plane trajectories of the transformed and original Chua's circuit. Fig. 15 clearly indicates the fact that the tracking performances are guaranteed by our hybrid adaptive FNN controller.

Fig. 16(a) shows the overall control effort $u$ for the first $6 \mathrm{~s}$. Fig. 16(b) extends the time-scale in Fig. 16(a) to 25 s. Obviously, the overall control effort $u$ in the steady state has its maximum magnitude less than 5 NT. Fig. 16(c) shows the supervisory control $u_{s}$ with its activation and activation periods in the initial $5 \mathrm{~s}$. After $5 \mathrm{~s}$, the $u_{s}$ is no longer necessary.

Applying a different weighting factor $\alpha$, the tracking error performance of Example 2 is shown in Fig. 17.
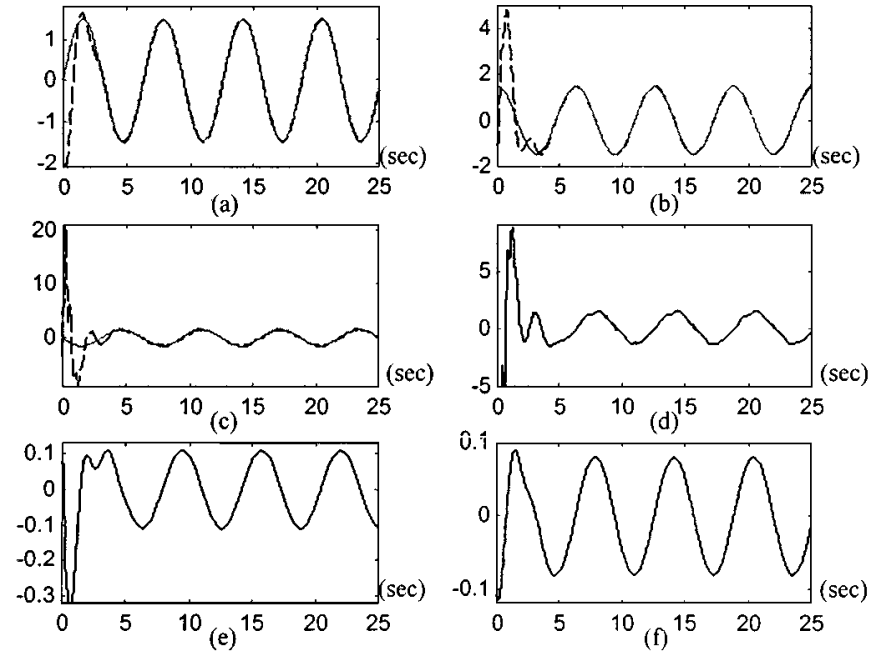

Fig. 14. (a) Output trajectories of $y$ (dashed line) and $y_{r}$ (solid line) with $\alpha=$ 0.9 . (b) Output trajectories of $\dot{y}$ (dashed line) and $\dot{y}_{r}$ (solid line) with $\alpha=0.9$. (c) Output trajectories of $\ddot{y}$ (dashed line) and $\ddot{y}_{r}$ (solid line) with $\alpha=0.9$. (d) Trajectory of $V_{C 1}$. (e) Trajectory of $V_{C 2}$. (f) Trajectory of $i_{L}$.

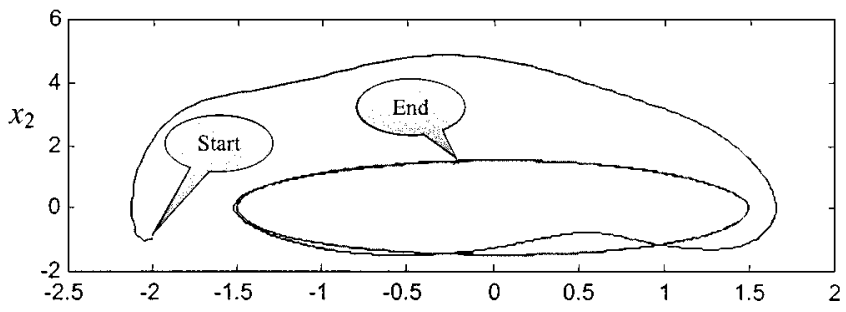

(a)

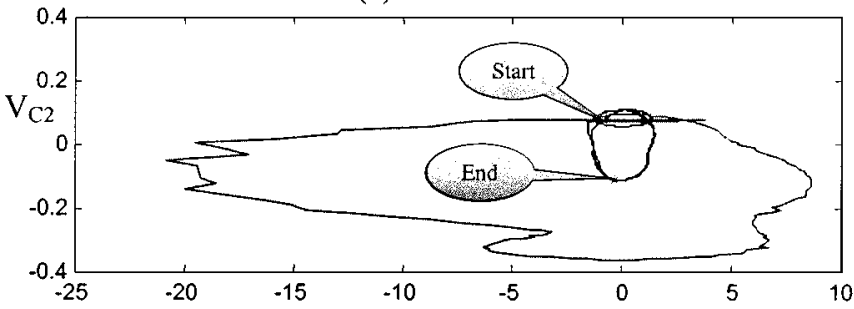

(b) $\mathrm{V}_{\mathrm{Cl}}$

Fig. 15. (a) Phase-plane trajectory of transformed Chua's circuit with $\alpha=$ 0.9. (b) Phase-plane trajectory of Chua's circuit.

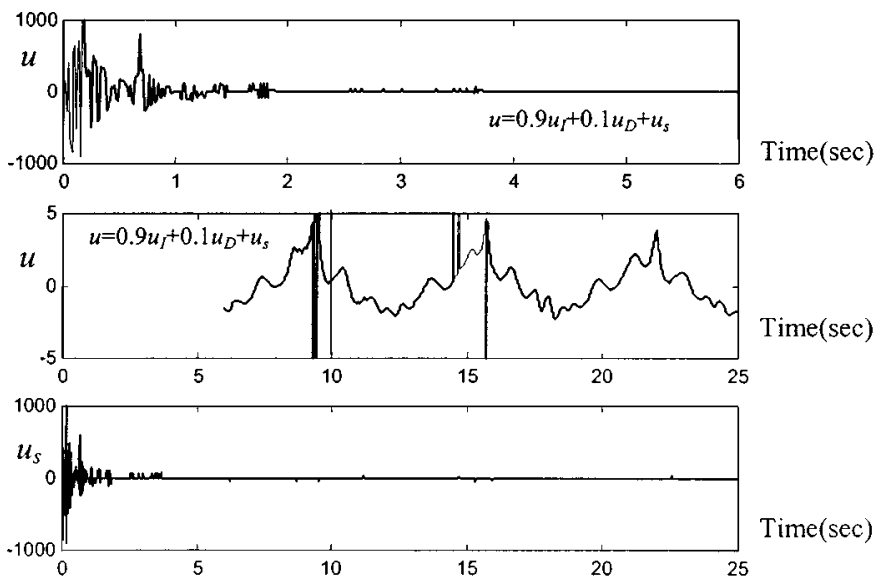

Fig. 16. (a) Trajectory of the control input (including supervisory control) with $\alpha=0.9$ (time $=0 \sim 6 \mathrm{~s}$ ). (b) Trajectory of the control input (including supervisory control) with $\alpha=0.9$ (time $=6 \sim 25 \mathrm{~s}$ ). (c) Trajectory of the supervisory control $u_{S}$ with $\alpha=0.9$. 


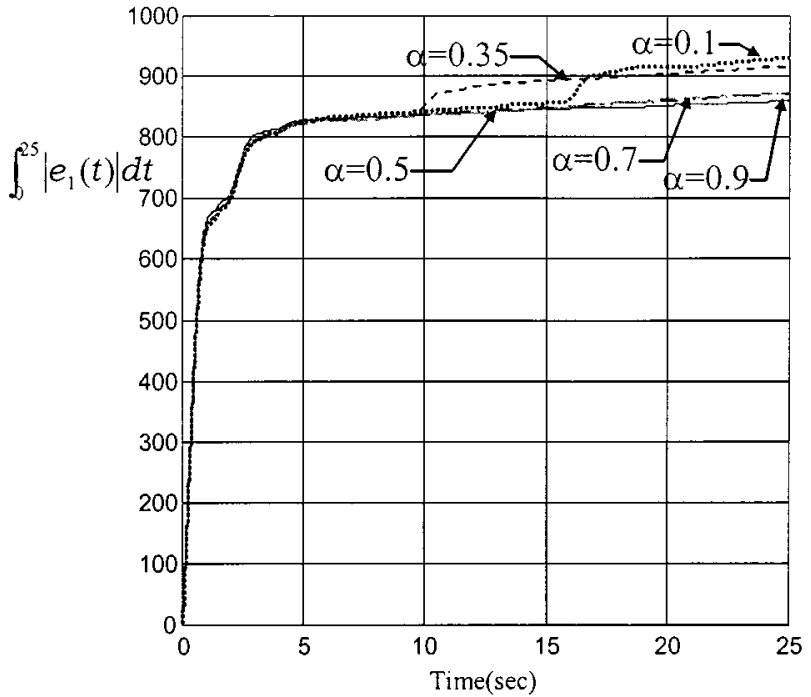

Fig. 17. Tracking performance $\int_{0}^{25} e_{1}^{2}(t) d t$ with different $\alpha$.

\section{CONCLUSION}

An observer-based hybrid direct/indirect adaptive FNN controller appended with a supervisory controller for a class of unknown nonlinear dynamical systems is proposed in this paper. It is a flexible design methodology by the tradeoff between plant knowledge and control knowledge using a weighting factor $\alpha$ adopted to sum together the control effort from indirect adaptive FNN controller and direct adaptive FNN controller. If the fuzzy descriptions of the plant are more important and viable, then choose large $\alpha$; otherwise, choose small $\alpha$. Based on the Lyapunov synthesis approach, the free parameters of the adaptive FNN controller can be tuned on-line by an observer-based output feedback control law and adaptive law. Furthermore, it is a valuable idea that the supervisory control is appended into the FNN controller. The supervisory controller will be activated to force the state to be within the constraint set as long as the system tends to be unstable controlled only by the FNN controller. On the other hand, if the FNN controller works well, the supervisory controller will be deactivated. The simulation results show explicitly that the tracking error of larger $\alpha$ is less than smaller $\alpha$ i.e., the plant knowledge is more important and viable, and the supervisory controller only works in the beginning period and after that the FNN controller is a main controller. Two nonlinear systems, namely, inverted pendulum system and Chua's chaotic circuit, are fully illustrated to track sinusoidal signals. Furthermore, it is obvious that the control effort is much less and tracking performance is better than those in previous works.

\section{REFERENCES}

[1] S. S. Sastry and A. Isidori, "Adaptive control of linearization systems," IEEE Trans. Automat. Contr., vol. 34, pp. 1123-1131, 1989.

[2] R. Marino and P. Tomei, "Globally adaptive output-feedback control on nonlinear systems, Part I: Linear parameterization," IEEE Trans. Automat. Contr., vol. 38, pp. 17-32, 1993.

[3] — "Globally adaptive output-feedback control on nonlinear systems-Part II: Nonlinear parameterization," IEEE Trans. Automat. Contr., vol. 38, pp. 33-48, 1993.

[4] B. S. Chen, C. H. Lee, and Y. C. Chang, " $H^{\infty}$ tracking design of uncertain nonlinear SISO systems: Adaptive fuzzy approach," IEEE Trans. Fuzzy Syst., vol. 4, pp. 32-43, Feb. 1996.
[5] G. A. Rovithakis and M. A. Christodoulou, "Adaptive control of unknown plants using dynamical neural networks," IEEE Trans. Syst., Man, Cybern., vol. 24, pp. 400-412, Mar. 1994.

[6] — " "Direct adaptive regulation of unknown nonlinear dynamical systems via dynamic neural networks," IEEE Trans. Syst., Man, Cybern., vol. 25, pp. 1578-1594, Dec. 1995.

[7] L. X. Wang, "Stable adaptive fuzzy control of nonlinear systems," IEEE Trans. Fuzzy Syst., vol. 1, pp. 146-155, May 1993.

[8] - Adaptive Fuzzy Systems and Control: Design and Stability Analysis. Englewood Cliffs, NJ: Prentice-Hall, 1994.

[9] J. T. Spooner and K. M. Passino, "Stable adaptive control using fuzzy systems and neural networks," IEEE Trans. Fuzzy Syst., vol. 4, pp. 339-359, Aug. 1996.

[10] X. J. Ma and Z. Q. Sun, "Output tracking and regulation of nonlinear system based on Takgi-Sugeno fuzzy model," IEEE Trans. Syst., Man, Cybern., vol. 30, pp. 47-59, Feb. 2000.

[11] A. S. Poznyak, W. Yu, E. N. Sanchez, and J. P. Perez, "Nonlinear adaptive tracking using dynamic neural networks," IEEE Trans. Neural Networks, vol. 10, pp. 1402-1411, Nov. 1999.

[12] Y. G. Leu, T. T. Lee, and W. Y. Wang, "Observer-based adaptive fuzzy neural control for unknown nonlinear dynamical systems," IEEE Trans. Syst., Man, Cybern., vol. 29, pp. 583-591, Oct. 1999.

[13] C. H. Wang, W. Y. Wang, T. T. Lee, and P. S. Tseng, "Fuzzy B-spline membership function (BMF) and its applications in fuzzy neural control," IEEE Trans. Syst., Man, Cybern., vol. 25, pp. 841-851, May 1995.

[14] Y. G. Leu, T. T. Lee, and W. Y. Wang, "Robust adaptive fuzzy neural controllers for uncertain nonlinear systems," IEEE Trans. Robot. Automat., vol. 15, pp. 805-817, Oct. 1999.

[15] J. L. Castro, "Fuzzy logical controllers are universal approximators," IEEE Trans. Syst., Man, Cybern., vol. 25, pp. 629-635, Apr. 1995.

[16] L. O. Chua, M. Komuro, and T. Matsumoto, "The double Scroll family: I and II," IEEE Trans. Circuit Syst., vol. CS-33, pp. 1072-1118, 1986.

[17] A. Isidori, Nonlinear Control Systems, 2nd ed. Berlin, Germany: Springer-Verlag, 1989.

[18] T. Takagi and M. Sugeno, "Fuzzy identification of systems and its applications to modeling and control," IEEE Trans. Syst., Man, Cybern., vol. SMC-15, pp. 116-132, Jan. 1985.

[19] M. Sugeno, "On stability of fuzzy systems expressed by fuzzy rules with singleton consequents," IEEE Trans. Fuzzy Syst., vol. 7, pp. 201-224, Apr. 1999.

[20] F. Cuesta, F. Gordillo, J. Aracil, and A. Ollero, "Stability analysis of nonlinear multivariable Takagi-Sugeno fuzzy control systems," IEEE Trans. Fuzzy Syst., vol. 7, pp. 508-519, Oct. 1999.

[21] M. C. M. Teixeira and S. H. Zak, "Stabilizing controller design for uncertain nonlinear systems using fuzzy models," IEEE Trans. Fuzzy Syst., vol. 7, pp. 133-142, Apr. 1999.

[22] O. Begovich, E. N. Sanchez, and M. Maldonado, "T-S scheme for trajectory tracking of an underactuated robot," in Proc. IEEE Fuzzy Conf., San Antonio, TX, May 2000, pp. 798-803.

[23] J. E. Slotine and W. Li, Applied Nonlinear Control. Englewood Cliffs, NJ: Prentice-Hall, 1991.

[24] Y. H. Joo, L. S. Shieh, and G. R. Chen, "Hybrid state space fuzzy model based controller with dual-rate sampling for digital control of chaotic systems," IEEE Trans. Fuzzy Syst., vol. 7, pp. 394-408, Aug. 1999.

[25] K. Ogata, Discrete-Time Control Systems. Englewood Cliffs, NJ: Prentice-Hall, 1987.

[26] C. T. Chen, Linear System Theory and Design, 3rd ed. London, U.K.: Oxford Univ. Press, 1999.

Chi-Hsu Wang (M'92-SM'93) was born in Tainan, Taiwan, R.O.C., in 1954. He received the B.S. degree in control engineering from the National Chiao-Tung University, Hsinchu, Taiwan, in 1976, the M.S. in computer science from the National Tsing-Hua University, Bejing, China, in 1978, and the Ph.D. degree in electrical and computer engineering from the University of Wisconsin, Madison, in 1986.

He was appointed Associate Professor in 1986, and Professor in 1990 in the Department of Electrical Engineering, National Taiwan University of Science and Technology, Taipei. He is currently with the School of Microelectronic Engineering, Griffith University, Nathan, Brisbane, Australia. His current research interests and publications are in the areas of digital control, FNN, intelligent control, adaptive control, and robotics. He has published over 35 international journal articles in these areas and has also served on program committees and chaired technical sessions for several international conferences. 
Tsung-Chih Lin was born in Taichung, Taiwan, R.O.C., in 1961. He received the B.S. degree in electrical engineering from the Feng-Chia University, Taichung, in 1984, and the M.S. degree in control engineering from the National Chiao-Tung University, Hsinchu, Taiwan, in 1986. He is currently pursuing the Ph.D. degree at the School of Microelectronic Engineering, Griffith University, Nathan, Brisbane, Australia.

He is currently a Lecturer at the Feng-Chia University. His current research interests and publications are in the areas of adaptive control, FNN, and robust control.

Tsu-Tian Lee (M'87-SM'89-F'97) was born in Taipei, Taiwan, R.O.C., in 1949. He received the B.S. degree in control engineering from the National Chiao-Tung University (NCTU), Hsinchu, Taiwan, in 1970, and the M.S. and $\mathrm{Ph} . \mathrm{D}$. degrees in electrical engineering from the University of Oklahoma, Norman, in 1972 and 1975, respectively.

In 1975, he was appointed Associate Professor and in 1978 Professor and Chairman of the Department of Control Engineering at NCTU. In 1981, he became Professor and Director of the Institute of Control Engineering, NCTU. In 1986, he was a Visiting Professor and in 1987, a Full Professor of Electrical Engineering at the University of Kentucky, Lexington. In 1990, he was a Professor and Chairman of the Department of Electrical Engineering at the National Taiwan University of Science and Technology (NTUST). In 1998, he became the Professor and Dean of the Office of Research and Development, NTUST. Since 2000, he has been with the Department of Electrical and Control Engineering, NCTU, where he is now a Chair Professor. He has published more than 170 refereed journal and conference papers in the areas of automatic control, robotics, fuzzy systems, and NNs. His current research involves motion planning, fuzzy and neural control, optimal control theory and application, and walking machines.

Prof. Lee received the Distinguished Research Award from the National Science Council, Taiwan, in 1991-1992, 1993-1994, 1995-1996, and 1997-1998, and the Academic Achievement Award in Engineering and Applied Science from the Ministry of Education, R.O.C., in 1998. He was elected to the grade of IEEE Fellow in 1997 and IEE Fellow in 2000. He became a Fellow of New York Academy of Sciences in 2002. His professional activities include serving on the Advisory Board of the Division of Engineering and Applied Science, National Science Council, serving as the Program Director, Automatic Control Research Program, National Science Council, and serving as an Advisor of Ministry of Education, Taiwan, as well as numerous consulting positions.
Han-Leih Liu was born in Taichung, Taiwan, R.O.C., in 1952. He received the B.S. degree in computer science from the Tam-Kang University, Taipei, Taiwan, in 1975, the M.S. degree in computer engineering from the City University of New York, NY, in 1985, and the Ph.D. degree from the School of Microelectronic Engineering, Griffith University, Nathan, Brisbane, Australia, in 2001

His current research interests and publications are in the areas of FNN and adaptive control. 